\title{
Ionic Homeostasis, Acid-base Balance and the Risk of Citrate Accumulation in Patients After Cardiovascular Surgery Treated With Continuous Veno-venous Haemofiltration With Post-dilution Regional Citrate Anticoagulation
}

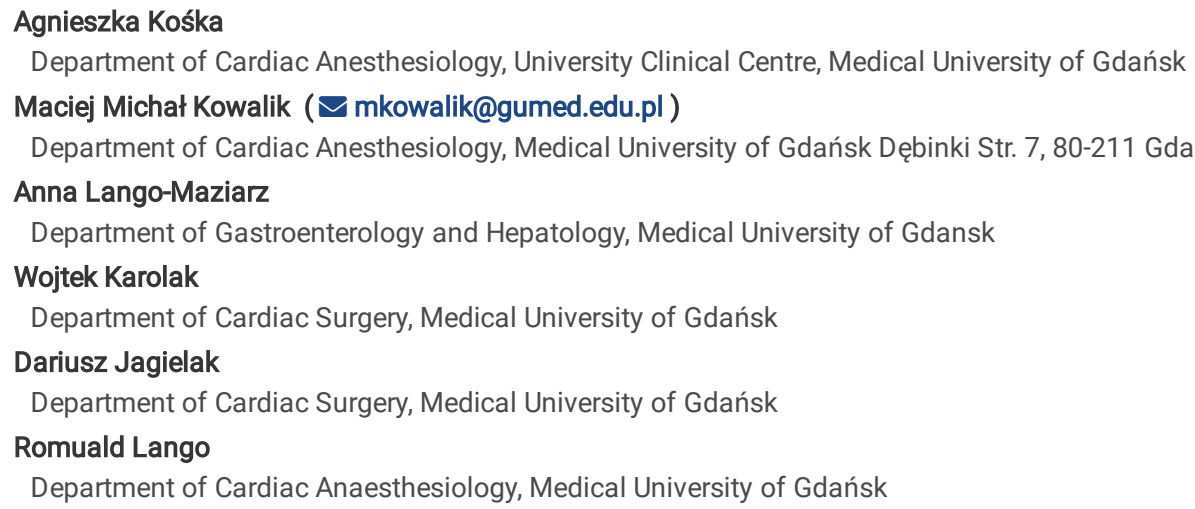




\section{Abstract}

Background: Patients with known or new-onset acute renal failure after cardiovascular surgery, requiring renal replacement therapy, can benefit from adequate non-heparin circuit anticoagulation. The idea behind not using heparin relates to the post-operative risk of bleeding. Simplified regional citrate anticoagulation (RCA) protocol proposes the use of citric acid dextrose formula A (ACD-A) during post-dilutional continuous veno-venous hemofiltration (CVVH) with standard bicarbonate buffered calcium containing replacement solution. Citrate accumulation diagnosed upon total to ionized calcium ratio (tCa/iCa) and low ionized calcium (iCa) are considered as the biggest risks related to regional citrate accumulation.

Methods: This prospective observational study evaluated electrolyte and acid-base homeostasis in cardiovascular surgery patients with known chronic or new-onset acute renal failure treated with post-dilution continuous veno-venous haemofiltration (CVVH) with a simplified RCA protocol with ACD-A. In total, 50 consecutive cardiovascular surgery patients treated with $\mathrm{CVVH}$ with RCA were evaluated. Base excess; $\mathrm{pH}$; bicarbonate, lactate, $\mathrm{Na}^{+}, \mathrm{Cl}^{-}, \mathrm{Mg}^{++}$, and inorganic phosphate concentrations; the total to ionized calcium ratio ( $\mathrm{tCa} / \mathrm{iCa})$; and high anion gap metabolic acidosis were assessed during haemofiltration treatment in survivors and non-survivors.

Results: Thirty-three (66\%) patients died. In total, 235 haemofiltration sessions with a median circuit survival time of 57 hours (1-117) were evaluated. The therapies were very well balanced with regard to sodium and chloride homeostasis. The lactate concentration and anion gap decreased during CVVH sessions longer than 72 hours, but no inter-group difference was observed. The tCa/iCa ratio exceeded 2.5 in 11 of 246 (4.5\%) readings and was significantly higher in non-survivors $(\mathrm{p}=0.037)$. No correlation was observed between the lactate concentration before haemofiltration and the tCa/iCa ratio during haemofiltration. Magnesium and phosphate concentrations decreased during CVVH, and additional supplementation with magnesium was necessary. The magnesium concentration was lower in the non-survivors.

Conclusions: The CVVH RCA protocol provides stable sodium and chloride concentrations and a tendency towards higher pH values and bicarbonate concentrations. Supplementation with magnesium and phosphate ions is needed. The incidence of citrate accumulation exceeded $4 \%$ and was significantly higher in non-survivors.

Trial registration: retrospectively registered: Clinicaltrials.gov, NCT03836742.

\section{Background}

The current cardiovascular surgery patient is relatively older, has multiple comorbidities and undergoes more complex surgical procedures; thus, acute kidney injury (AKI) is becoming an increasingly common complication after cardiac surgery, with more patients needing renal replacement therapy. AKI after cardiac surgery affects $2-5 \%$ of patients and has a mortality rate between 36 and $78 \%$ [1]. In patients with significant pre-operative risk factors, patients with preoperative or postoperative cardiogenic shock and in patients in whom cardiopulmonary bypass results in a severe inflammatory response, acute renal failure can develop, requiring continuous renal replacement therapy (CRRT) in the early or immediate postoperative hours. Due to the increased risk of postoperative bleeding, heparin anticoagulation is contraindicated in the early hours after operation, leaving regional citrate anticoagulation (RCA) as the best option for anticoagulation. According to the KDIGO guidelines, even with a lack of a contraindication for heparin, RCA should be selected rather than heparin anticoagulation, as it is related to increased filter life-span, a lower incidence of bleeding and better metabolic control [2,3]. Although citrate anticoagulation is considered superior for preventing filter clotting and clogging, it carries the risk of several complications, including acid-base abnormalities, hypocalcaemia, hypernatremia and hypomagnesemia $[4,5]$.

Disturbed liver function and tissue hypoperfusion, as manifested by increased lactate levels, are common in cardiac surgery patients, rendering citrate metabolism less predictable than in the non-cardiac surgical intensive care setting. Hypocalcaemia might more readily exacerbate heart failure after cardiac surgery patients than in the general ICU population [6]. Decreased citrate metabolism in the liver and muscles can result in citrate accumulation (CA). Because citrate metabolism is oxygen dependent, shock and tissue hypoperfusion are related to impaired metabolism. In contrast, an overdose of citrate in the case of properly functioning citrate metabolism may lead to the development of metabolic alkalosis [5].

Citrate exerts its anticoagulation effect by chelating calcium ions, which should decrease their concentration below $0.4 \mathrm{mmol} / \mathrm{L}$ in the CRRT circuit. A great deal of calcium ions bound to citrate are filtered out and must be replaced by the continuous infusion of calcium chloride or calcium gluconate before the blood is returned to the patient. Due to the lack of commercially available tests of the serum citrate concentration, the assessment of the ratio of the total to ionized calcium concentrations ( $\mathrm{tCa} / \mathrm{iCa}$ ) and high anion gap metabolic acidosis (HAGMA) remain the only feasible methods for the clinical assessment of $\mathrm{CA}$ [7]. An increase in the tCa/iCa ratio exceeding 2.5 is related to a high risk of citrate accumulation, and if this ratio exceeds 3.0 , CA can be diagnosed [8].

When implementing a novel CRRT protocol with dedicated substitution fluids and anticoagulation algorithms, physicians expect that the method provides the desired electrolyte and acid-base balance. However, companies providing CRRT equipment and fluids do not guarantee that the proposed treatment protocols will correct electrolyte imbalances or acid-base abnormalities. In most studies on CRRT in ICU patients, the assessment of the safety of the therapy is limited to the incidences of bleeding, arrhythmia, and haemodynamic instability. We strongly believe that the currently available blood purification protocols should also be evaluated with respect to their influence on $\mathrm{pH}$ and electrolyte imbalances, which are known risk factors for mortality and morbidity in the intensive care setting $[9,10]$. There have been a few studies that have focused on the incidence of acid-base imbalance and dyselectrolytaemia during CRRT with RCA [11-13]. The risk of electrolyte disorders and acid-base imbalance should be clinically evaluated for every novel protocol proposed for use in clinical practice. To the best of our knowledge, the simplified citrate anticoagulation protocol for post-dilution continuous veno-venous (CVVH) haemofiltration described by Kirwan et al. has not been evaluated with respect to ion and acid-base homeostasis in cardiovascular surgery patients with acute renal failure [14].

\section{Materials And Methods}


The primary objective of this study was to compare the incidence of eventual electrolyte and acid-base disorders between survivors and non-survivors during post-dilution $\mathrm{CVVH}$ treatment with a simplified anticoagulant citrate dextrose solution A (ACD-A)-based RCA protocol in cardiovascular surgery patients. The secondary aim was to monitor the risk of CA during RCA haemofiltration treatment.

We conducted a prospective single-centre observational study with all consecutive patients treated with CVVH RCA between September 2015 and November 2017 in a cardiovascular surgery ICU.

All patients requiring renal replacement therapy and treated with CVVH RCA were enrolled in the study, including patients with end-stage renal failure treated with intermittent haemodialysis before surgery. The decision to start renal replacement therapy was made by treating intensivists based on the clinical features of volume overload and biochemical features of azotaemia or dyselectrolytaemia [15]. Contraindications to RCA were severe chronic liver disease, acute liver injury with an prothrombin time international normalized ratio (INR) $>2$ and refractory shock with a lactate level above $8 \mathrm{mmol} / \mathrm{L}$. The most recent serum creatinine concentration before surgery was considered the baseline value. The recorded CRRT parameters included blood and filtrate flows, citrate and calcium chloride solution flows and filter life-span.

\section{Haemofiltration and RCA protocol}

CVVH was performed with the Aquarius + CRRT device with version 6.02.14/15 software (Aquarius system, NIKKISO Europe GmbH, Desbrocksriede 1, 30855 Langenhagen, Germany) and Citraset RCA for therapies with regional citrate anticoagulation comprising Aqualine RCA (Haemotronic S.p. A, Via Carreri 16, 41037 Mirandola, Italy) and the Aquamax haemofilter (Nikkiso Belgium bvba, Industriepark 6, 3300 Tienen, Belgium). In most cases, the filter size (either Aquamax $1.2 \mathrm{~m}^{2}$ or Aquamax $1.9 \mathrm{~m}^{2}$ ) was determined by the treating clinician, the patient's actual body weight (>90 kg) and symptoms of distributive shock requiring noradrenaline infusion over $0.1 \mu \mathrm{g} / \mathrm{kg} / \mathrm{hour}$, in which case the Aquamax 19 filter was used. In all patients, Accusol $35 \mathrm{KO}$ (Nikkiso, Belgium Industriepark 6 B-3300 Tienen; Belgium), which contains $1.75 \mathrm{mmol} / \mathrm{L}$ of calcium, was used as the post-dilution replacement fluid. All substitution solution bags were supplemented with potassium to reach a $4 \mathrm{mEq} / \mathrm{L}$ concentration. Anticoagulant citrate dextrose solution A, U.S.P. (ACD-A) (ACD-A, Macopharma, 5003F Rue Lorthiois, 59420 Mouvaux, France) was used as the source of citrate. Three thousand units of UFH were added to 1 litre $0.9 \% \mathrm{NaCl}$ solution for CRRT circuit priming in all but 7 patients who were suspected of having heparin-induced thrombocytopenia. The median circuit life-span was 57 hours (range: $\left.1-117, Q_{1}=27, Q_{3}=83\right)$, and it did not differ between survivors and non-survivors. The following CVVH parameters were recorded at the beginning of the CVVH session: blood flow $187.4 \pm 34.8 \mathrm{~mL} / \mathrm{min}$ (80-300), replacement fluid flow $2213 \pm 383 \mathrm{~mL} / \mathrm{h}(1300-2700)$, ultrafiltration $234 \pm 90 \mathrm{~mL} / \mathrm{h}$ (10-500), citrate flow $279 \pm 53.1 \mathrm{~mL} / \mathrm{h}(104-350)$, calcium flow $78.5 \pm 44.5 \mathrm{~mL} / \mathrm{h}(0-200)$, and dialysis dose $32.1 \pm 5.4 \mathrm{ml} / \mathrm{kg} / \mathrm{h}(18.4-50)$.

The initial settings of the haemofiltration parameters and their modifications when metabolic alkalosis was observed were adopted from the CVVH RCA protocol published by Kirwan et al.; however, the renal dose was calculated for the actual body weight [14]. The prescribed starting CRRT dosage was 20$25 \mathrm{ml} / \mathrm{kg} / \mathrm{h}$ and was adjusted according to the desired urea clearance. Ultra-filtration was titrated by the treating physician according to clinical indications and individual patient's haemodynamic performance. The plasma concentration of ionized calcium was supplemented with additional calcium using an initial dose of $10 \mathrm{~mL}$ of $10 \%$ calcium chloride (WZF Polfa, Karolkowa Str. 22/24, Warsaw, Poland) added to $1 \mathrm{~L}$ of normal saline, which resulted in a Ca++ concentration of $4.6 \mathrm{mmol} / \mathrm{L}$. We observed a low iCa plasma concentration in the first 10 patients, and this dose was increased to $20 \mathrm{~mL}$ of $10 \%$ calcium chloride. After the following 10 patients, $40 \mathrm{~mL}$ of $10 \%$ calcium chloride yielded a Ca+ ${ }^{++}$concentration of $18.4 \mathrm{mmol} / \mathrm{L}$. The target calcium concentration in the plasma was increased from the initial 0.9-1.2 range to the 1.0-1.2 range. Furthermore, the original protocol was modified by the additional routine infusion of magnesium sulfate at a fixed rate of $0.2 \mathrm{~g} / \mathrm{hour}(0.81 \mathrm{mmol} / \mathrm{hour})$. Blood flow was set to achieve a filtration ratio of $\sim 20 \%$. If the plasma $\mathrm{pH}$ increased above 7.5 or the bicarbonate concentration was above $40 \mathrm{mmol} / \mathrm{L}$, the haemofiltration dose was decreased from the initial $35 \mathrm{~mL} / \mathrm{kg} / \mathrm{hour}$ to $25 \mathrm{~mL} / \mathrm{kg} / \mathrm{hour}$. If metabolic alkalosis persisted, the citrate dose was reduced, and if this did not resolve the issue within 3 hours, RCA was stopped [14].

\section{Details of the anticoagulation therapy}

Six patients received no antithrombotic medication or acetylsalicylic acid (ASA) alone, 15 patients received a prophylactic dose of low molecular weight heparin (LMWH) or fondaparinux, with or without ASA, twenty-eight patients received a therapeutic dose of LMWH or fondaparinux with or without ASA, and 3 patients were treated with continuous unfractionated heparin infusion. The detailed analysis of filter life-span with regard to anticoagulation methods in this case series were published elsewhere [16].

\section{Outcome measures}

According to the reference values used by the hospital laboratory, the following parameters were adopted for the diagnosis of electrolyte disorders: hyponatraemia $-\mathrm{Na}^{+}<135 \mathrm{mEq} / \mathrm{L}$, hypernatraemia $-\mathrm{Na}^{+}>145 \mathrm{mEq} / \mathrm{L}$, hypochloraemia $-\mathrm{Cl}^{-}<98 \mathrm{mEq} / \mathrm{L}$, hyperchloraemia $-\mathrm{Cl}^{-}>112 \mathrm{mEq} / \mathrm{L}$, hypocalcaemia iCa $<0.98 \mathrm{mmol} /$, hypercalcaemia - iCa $>1.21 \mathrm{mmol} / \mathrm{L}$, hypomagnesaemia - $\mathrm{Mg}^{++}<1.5 \mathrm{mg} / \mathrm{dL}$, and hypophosphataemia $-\mathrm{PO}_{4}{ }^{3+}<2.3 \mathrm{mg} / \mathrm{dL}$. Severe hypernatraemia was diagnosed when $\mathrm{Na}^{+}$was $>150 \mathrm{mEq} / \mathrm{L}$, severe hypochloraemia was diagnosed when $\mathrm{Cl}^{-}$was $<92 \mathrm{mEq} / \mathrm{L}$, severe hypocalcaemia was diagnosed when iCa was $<0.9 \mathrm{mmol} / \mathrm{L}$, and severe hypercalcaemia was diagnosed when iCa was $>1.32 \mathrm{mmol} / \mathrm{L}$. The incidence of $\mathrm{tCa} / \mathrm{iCa}>2.5$, as the parameter indicating the risk of CA, was reported. Ionized calcium was not corrected for the albumin concentration [17]. Metabolic alkalosis was diagnosed based on a $\mathrm{pH}>7.45$ and $\mathrm{HCO}_{3}{ }^{-}$concentration $>26 \mathrm{mmol} / \mathrm{L}$. $\mathrm{HAGMA}$ was reported if the high anion gap (calculated as $\mathrm{Na}^{+}-\left(\mathrm{Cl}^{-}+\mathrm{HCO}_{3}{ }^{-}\right)$) exceeded 12 and the bicarbonate concentration was lower than $20 \mathrm{mmol} / \mathrm{L}$. CA was diagnosed when at least three out of the following four systemic metabolic diagnostic criteria were present: 1) a decrease in systemic ionized calcium to a value below $1.1 \mathrm{mmol} / \mathrm{L}, 2$ ) an increase of tCa/iCa to a value $>2.25,3$ ) metabolic acidosis $(\mathrm{pH}<7.2$ or $\mathrm{BE}<-5 \mathrm{mmol} / \mathrm{L})$ and 4$)$ an anion gap greater than $12 \mathrm{mmol} / \mathrm{L}$.

\section{Laboratory analyses}


Creatinine, blood urea nitrogen (BUN), magnesium, tCa, and phosphate were measured once a day. Arterial blood gas analysis, sodium, potassium, chloride, iCa, bicarbonate, base excess, anion gap, lactate and haemoglobin were assessed every 6 hours. Blood gas analyses were performed on an ABL800 Flex 835 blood analyser (Radiometer, Copenhagen, Denmark). All the exams performed were part of the routine post-operative ICU protocol. The total calcium concentration was assessed with the Arsenazo III method (Abbott Laboratories Diagnostics Division, Abbott Park, IL, USA). The magnesium ion concentration was assessed with the isothiocyanate dehydrogenase enzymatic method (Abbott Laboratories Diagnostics Division, Abbott Park, IL, USA). The inorganic phosphate serum concentration was assessed by a colorimetric method based on phosphomolybdic acid reduction to molybdenum blue. Magnesium, tCa and inorganic phosphate concentrations were adopted for the analysis if the time span between blood sampling and the time point of the study (which was related to the beginning of $\mathrm{CVVH}$ treatment) was less than 6 hours. For the purpose of the analyses of the stability of the sodium, chloride, ionized calcium, $\mathrm{pH}$, bicarbonate and lactate concentrations, data from sessions lasting at least 72 hours were selected.

\section{Statistical analysis}

Data were tested for the normality of their distributions using the Shapiro-Wilk test. All baseline variables are presented using descriptive summary statistics such as the means \pm standard deviation (SD) or medians with quartiles and 25th and 75th interquartile ranges (IQR), as appropriate. Normally distributed data were compared using Student's t-tests; nonnormally distributed data were compared with the Mann-Whitney U test. Categorical variables are expressed as proportions and were compared between groups using the $\chi^{2}$ test or Fisher's two-tailed exact test depending on the sample size. Comparisons between multiple sets of measured parameters were performed with repeated-measures ANOVA and are presented as the means \pm SDs. Statistical analyses were performed with Statistica 10 software (StatSoft Inc., Tulsa, USA). Statistical significance was set at the 0.05 level.

\section{Results}

\section{Patient characteristics}

During the study period, 54 cardiovascular surgery patients were treated with CVVH RCA. Four patients were excluded from the analysis due to reasons provided in the patient flow chart (Fig. 1). The 50 patients underwent a total of 233 haemofiltration treatment sessions with RCA that were analysed. Patient characteristics and cardiovascular surgical procedures are detailed in Table 1 (Table 1). 
Table 1

Patient characteristics and type of surgical procedures in $n=52$ patients after cardiovascular surgery who were treated with renal replacement therapy with regional citrate anticoagulation.

\begin{tabular}{|c|c|c|c|c|}
\hline Patient characteristics/type of surgery & Total & $\begin{array}{l}\text { Survivors } \\
N=17\end{array}$ & $\begin{array}{l}\text { Non-survivors } \\
\mathrm{N}=35\end{array}$ & p \\
\hline Age, years & $\begin{array}{l}70\left(Q_{1}=62, Q_{3}=74 ; \text { Range: } 37-\right. \\
84)\end{array}$ & $\begin{array}{l}64\left(Q_{1}=57.2, Q_{3}=70 ; \text { Range: }\right. \\
37-78)\end{array}$ & $\begin{array}{l}72\left(Q_{1}=65, Q_{3}=76 ; \text { Range: }\right. \\
48-84)\end{array}$ & 0.029 \\
\hline $\mathrm{BMI}, \mathrm{kg} / \mathrm{m}^{2}$ & $\begin{array}{l}27.9\left(Q_{1}=24.2, Q_{3}=31.6 ; \text { Range: }\right. \\
17-31.6)\end{array}$ & $\begin{array}{l}27.5\left(Q_{1}=23.6, Q_{3}=31.2\right. \\
\text { Range: } 20-36)\end{array}$ & $\begin{array}{l}28\left(Q_{1}=25.7, Q_{3}=33.4 ; \text { Range: }\right. \\
17-45)\end{array}$ & 0.161 \\
\hline Sex (male) & 28 & $9(53 \%)$ & $19(54 \%)$ & 0.93 \\
\hline Pre-existing renal disease & 25 & $7(41 \%)$ & $18(51 \%)$ & 0.488 \\
\hline Chronic dialysis treatment & 4 & $2(12 \%)$ & $2(6 \%)$ & 0.589 \\
\hline Diabetes mellitus & 12 & $3(18 \%)$ & $9(26 \%)$ & 0.729 \\
\hline Arterial hypertension & 33 & $9(53 \%)$ & $24(69 \%)$ & 0.272 \\
\hline COPD & 4 & $1(6 \%)$ & $3(9 \%)$ & 1 \\
\hline Hyperthyroidism & 3 & $3(18 \%)$ & $0(0 \%)$ & 0.031 \\
\hline Hypothyroidism & 4 & $1(6 \%)$ & $3(9 \%)$ & 1 \\
\hline Chronic atrial fibrillation & 11 & $2(12 \%)$ & $9(26 \%)$ & 0.304 \\
\hline Pre-operative haemoglobin [g/dL] & $\begin{array}{l}11.3 \\
\left(Q_{1}=9.8, Q_{3}=12.5 ; \text { Range: } 7.2-\right. \\
16.3)\end{array}$ & $\begin{array}{l}11.7 \\
\left(Q_{1}=10.5, Q_{3}=13.6 ; \text { Range: } 9.0-\right. \\
16.3)\end{array}$ & $\begin{array}{l}10.9 \\
\left(Q_{1}=9.4, Q_{3}=12.2 ; \text { Range: }\right. \\
7.2-13.9)\end{array}$ & 0.11 \\
\hline Pre-operative haemoglobin < 10 [g/dL] & 16 & $3(18 \%)$ & $13(37 \%)$ & 0.208 \\
\hline $\begin{array}{l}\text { Pre-operative creatinine concentration } \\
\text { [mg/dL] }\end{array}$ & $\begin{array}{l}1.49 \\
\left(Q_{1}=1.04, Q_{3}=2.10 ; \text { Range }\right. \\
0.62-9.31)\end{array}$ & $\begin{array}{l}1.49 \\
\left(Q_{1}=1.02, Q_{3}=2.02 ; \text { Range }\right. \\
0.61-6.15)\end{array}$ & $\begin{array}{l}1.5 \\
\left(Q_{1}=0.89, Q_{3}=2.5 ; \text { Range }\right. \\
0.69-9.3)\end{array}$ & 0.923 \\
\hline TYPE OF SURGERY & & & & \\
\hline Valvular surgery & 21 & 6 & 15 & 0.60 \\
\hline $\begin{array}{l}\text { Revascularization surgery (CABG and } \\
\text { OPCABG) }\end{array}$ & 7 & 4 & 3 & 0.20 \\
\hline Valvular + revascularization & 7 & 1 & 6 & 0.40 \\
\hline Other cardiac surgery & 2 & 0 & 2 & 1.00 \\
\hline $\begin{array}{l}\text { Vascular surgery including thoracic } \\
\text { aorta surgery }\end{array}$ & 10 & 3 & 7 & 1.00 \\
\hline Extracorporeal support (ECMO/VAD) & 2 & 1 & 1 & 1.00 \\
\hline Heart transplant & 1 & 1 & 0 & 0.32 \\
\hline Pericardial drainage & 1 & 0 & 1 & 1.00 \\
\hline PM electrodes removal & 1 & 1 & 0 & 0.32 \\
\hline
\end{tabular}

Of the 50 analysed patients, 33 (66.0\%) died before discharge from the hospital. Patient age was significantly higher in non-surviving group than in the surviving group. The incidence of hyperthyroidism was higher among survivors. No other significant difference was observed between survivors and nonsurvivors among the analysed pre-operative parameters. Among 48 patients who were not treated with intermittent haemodialysis before surgery, renal function recovered in 14 patients (29.1\%), 10 of whom were survivors (66.7\%) and 4 of whom were ultimately non-survivors (12.1\%).

\section{Ionic homeostasis}

Any kind of dyselectrolytemia before the beginning of the haemofiltration session was observed in 248 of a total of 946 ( $26.2 \%)$ electrolyte analyses. After 24 , 48 and 72 hours of haemofiltration, dyselectrolytemia was observed in 136 of 804 (16.9\%), 121 of 601 (20.1\%), and 79 of 400 (19.8\%) readings, respectively. The only difference between groups in the incidence of electrolyte disorders was that hyperchloraemia before the commencement of $\mathrm{CVVH}$ treatment was more common in survivors than in non-survivors. Hypernatraemia during $\mathrm{CVVH}$ was observed in 10 out of 424 measurements (2.4\%). The incidences of hyponatraemia, hypernatraemia, severe hypernatraemia, hypochloraemia and hyperchloraemia were significantly lower after 24,48 and 72 hours of $\mathrm{CVVH}$ 
treatment than the baseline value. The incidences of hyperchloraemia and hypernatraemia before the beginning of haemofiltration and the incidence of hypochloraemia after 24 hours of haemofiltration were significantly higher in survivors than in non-survivors.

Out of 233 haemofiltration sessions, 94 (40\%) lasted longer than 72 hours. The incidence of specific electrolytic and acid-base disorders in survivors and nonsurvivors is presented in Table 2 (Table 2).

Table 2

The incidences of electrolytic and acid-base disorders during $n=232$ haemofiltration sessions performed in $n=50$ patients. Data are presented as cases/all $b$ / time-point. (\# $p<0.01$ for difference between groups, \#\# $p<0.05$ for difference between groups, * $p<0.01$ for difference from the value before CVVH, ** $p<0.0$

\begin{tabular}{|c|c|c|c|c|c|c|c|c|c|c|c|}
\hline & \multicolumn{3}{|l|}{ Start HF } & \multicolumn{3}{|c|}{24 hours HF } & \multicolumn{3}{|c|}{48 hours HF } & \multicolumn{2}{|c|}{72 hours HF } \\
\hline & Survivors & $\begin{array}{l}\text { Non- } \\
\text { survivors }\end{array}$ & All & Survivors & $\begin{array}{l}\text { Non- } \\
\text { survivors }\end{array}$ & All & Survivors & $\begin{array}{l}\text { Non- } \\
\text { survivors }\end{array}$ & All & Survivors & $\begin{array}{l}\text { Non- } \\
\text { surviv }\end{array}$ \\
\hline $\begin{array}{l}\text { Hyponatremia } \\
(\mathrm{Na}<135 \mathrm{mEq} / \mathrm{L})\end{array}$ & $\begin{array}{l}12 / 54 \\
(22.2 \%)\end{array}$ & $\begin{array}{l}45 / 178 \\
(25.3 \%)\end{array}$ & $\begin{array}{l}57 / 232 \\
(24.6 \%)\end{array}$ & $\begin{array}{l}6 / 41 \\
(14.6 \%)\end{array}$ & $\begin{array}{l}10 / 148 \\
(6.8 \%)\end{array}$ & $\begin{array}{l}16 / 189 * \\
(8.5 \%)\end{array}$ & $\begin{array}{l}1 / 29 \\
(3.4 \%)\end{array}$ & $\begin{array}{l}4 / 112 \\
(3.6 \%)\end{array}$ & $\begin{array}{l}5 / 141 * \\
(3.5 \%)\end{array}$ & $\begin{array}{l}1 / 22 \\
(4.5 \%)\end{array}$ & $\begin{array}{l}1 / 72 \\
(1.4 \%\end{array}$ \\
\hline $\begin{array}{l}\text { Hypernatremia } \\
(\mathrm{Na}>145 \mathrm{mEq} / \mathrm{L})\end{array}$ & $\begin{array}{l}\text { 10/54\#\# } \\
(18.5 \%)\end{array}$ & $\begin{array}{l}\text { 13/178\#\# } \\
(7.3 \%)\end{array}$ & $\begin{array}{l}23 / 232 \\
(9.9 \%)\end{array}$ & $\begin{array}{l}2 / 41 \\
(4.9 \%)\end{array}$ & $\begin{array}{l}2 / 148 \\
(1.4 \%)\end{array}$ & $\begin{array}{l}\text { 4/189* } \\
\text { (2.1\%) }\end{array}$ & $\begin{array}{l}1 / 29 \\
(3.4 \%)\end{array}$ & $\begin{array}{l}3 / 112 \\
(2.7 \%)\end{array}$ & $\begin{array}{l}4 / 141 * * \\
(2.8 \%)\end{array}$ & $\begin{array}{l}1 / 22 \\
(4.5 \%)\end{array}$ & $\begin{array}{l}1 / 72 \\
(1.4 \%\end{array}$ \\
\hline $\begin{array}{l}\text { Severe } \\
\text { hypernatremia } \\
(\mathrm{Na}>150 \mathrm{mEq} / \mathrm{L})\end{array}$ & $\begin{array}{l}4 / 54 \\
(7.4 \%)\end{array}$ & $\begin{array}{l}7 / 178 \\
(3.9 \%)\end{array}$ & $\begin{array}{l}11 / 232 \\
(4.7 \%)\end{array}$ & $\begin{array}{l}1 / 41 \\
(2.4 \%)\end{array}$ & $\begin{array}{l}0 / 148 \\
(0 \%)\end{array}$ & $\begin{array}{l}1 / 189 * * \\
(0.5 \%)\end{array}$ & $\begin{array}{l}0 / 29 \\
(0 \%)\end{array}$ & $\begin{array}{l}1 / 112 \\
(0.9 \%)\end{array}$ & $\begin{array}{l}1 / 141 * * \\
(0.7 \%)\end{array}$ & $\begin{array}{l}0 / 22 \\
(0 \%)\end{array}$ & $\begin{array}{l}0 / 72 \\
(0 \%)\end{array}$ \\
\hline $\begin{array}{l}\text { Hypochloremia } \\
(\mathrm{Cl}<98 \mathrm{mEq} / \mathrm{L})\end{array}$ & $\begin{array}{l}10 / 54 \\
(18.5 \%)\end{array}$ & $\begin{array}{l}24 / 177 \\
(13.6 \%)\end{array}$ & $\begin{array}{l}34 / 231 \\
(14.7 \%)\end{array}$ & $\begin{array}{l}8 / 41 \# \\
(19.5 \%)\end{array}$ & $\begin{array}{l}8 / 148 \# \\
(5.4 \%)\end{array}$ & $\begin{array}{l}16 / 189 * * \\
(8.5 \%)\end{array}$ & $\begin{array}{l}3 / 29 \\
(10.3 \%)\end{array}$ & $\begin{array}{l}8 / 111 \\
(7.2 \%)\end{array}$ & $\begin{array}{l}11 / 140 * * \\
(7.9 \%)\end{array}$ & $\begin{array}{l}4 / 22 \\
(18.1 \%)\end{array}$ & $\begin{array}{l}6 / 72 \\
(8.3 \%\end{array}$ \\
\hline $\begin{array}{l}\text { Severe } \\
\text { hypochloremia } \\
(\mathrm{Cl}<92 \mathrm{mEq} / \mathrm{L})\end{array}$ & $\begin{array}{l}2 / 54 \\
(3.7 \%)\end{array}$ & $\begin{array}{l}3 / 177 \\
(1.7 \%)\end{array}$ & $\begin{array}{l}5 / 231 \\
(2.2 \%)\end{array}$ & $\begin{array}{l}0 / 41 \\
(0 \%)\end{array}$ & $\begin{array}{l}0 / 148 \\
(0 \%)\end{array}$ & $\begin{array}{l}0 / 189 \\
(0 \%)\end{array}$ & $\begin{array}{l}0 / 29 \\
(0 \%)\end{array}$ & $\begin{array}{l}0 / 111 \\
(0 \%)\end{array}$ & $\begin{array}{l}0 / 140 \\
(0 \%)\end{array}$ & $\begin{array}{l}0 / 22 \\
(0 \%)\end{array}$ & $\begin{array}{l}0 / 72 \\
(0 \%)\end{array}$ \\
\hline $\begin{array}{l}\text { Hyperchloremia } \\
(\mathrm{Cl}>107 \text { mEq/L) }\end{array}$ & $\begin{array}{l}16 / 54 \# \\
(29.6 \%)\end{array}$ & $\begin{array}{l}\text { 15/177\# } \\
(8.5 \%)\end{array}$ & $\begin{array}{l}31 / 231 \\
(13.4 \%)\end{array}$ & $\begin{array}{l}1 / 41 \\
(7.1 \%)\end{array}$ & $\begin{array}{l}4 / 148 \\
(2.7 \%)\end{array}$ & $\begin{array}{l}5 / 189 * \\
(2.6 \%)\end{array}$ & $\begin{array}{l}3 / 29 \\
(10.3 \%)\end{array}$ & $\begin{array}{l}4 / 111 \\
(3.6 \%)\end{array}$ & $\begin{array}{l}7 / 140 * \\
(5 \%)\end{array}$ & $\begin{array}{l}1 / 22 \\
(4.5 \%)\end{array}$ & $\begin{array}{l}2 / 72 \\
(2.8 \%\end{array}$ \\
\hline $\begin{array}{l}\text { Hypomagnesemia } \\
(\mathrm{Mg}<1.5 \mathrm{mg} / \mathrm{dL})\end{array}$ & $\begin{array}{l}7 / 27 \\
(25.9 \%)\end{array}$ & $\begin{array}{l}15 / 111 \\
(13.5 \%)\end{array}$ & $\begin{array}{l}22 / 138 \\
(15.9 \%)\end{array}$ & $\begin{array}{l}\text { 8/28\#\# } \\
(28.6 \%)\end{array}$ & $\begin{array}{l}\text { 13/101\#\# } \\
(12.9 \%)\end{array}$ & $\begin{array}{l}21 / 129 \\
(16.3 \%)\end{array}$ & $\begin{array}{l}9 / 21 \\
(42.9 \%)\end{array}$ & $\begin{array}{l}18 / 73 \\
(24.6 \%)\end{array}$ & $\begin{array}{l}27 / 94 * * \\
(28.7 \%)\end{array}$ & $\begin{array}{l}6 / 14 \\
(42.9 \%)\end{array}$ & $\begin{array}{l}10 / 5 C \\
(20 \%)\end{array}$ \\
\hline $\begin{array}{l}\text { Hypophosphatemia } \\
(\mathrm{Pi}<2.3 \mathrm{mg} / \mathrm{dL})\end{array}$ & $\begin{array}{l}3 / 19 \\
(15.8 \%)\end{array}$ & $\begin{array}{l}14 / 94 \\
(14.9 \%)\end{array}$ & $\begin{array}{l}17 / 113 \\
(15 \%)\end{array}$ & $\begin{array}{l}13 / 24 \# \\
(54.2 \%)\end{array}$ & $\begin{array}{l}14 / 84 \# \\
(16.7 \%)\end{array}$ & $\begin{array}{l}27 / 108 \\
(25 \%)\end{array}$ & $\begin{array}{l}11 / 18 \# \\
(61.1 \%)\end{array}$ & $\begin{array}{l}18 / 67 \# \\
(26.7 \%)\end{array}$ & $\begin{array}{l}29 / 85^{\star} \\
(34.1 \%)\end{array}$ & $\begin{array}{l}13 / 14 \# \\
(92.9 \%)\end{array}$ & $\begin{array}{l}16 / 4 C \\
(40 \%)\end{array}$ \\
\hline $\begin{array}{l}\text { Hypocalcemia (iCa } \\
<0.98 \mathrm{mmol} / \mathrm{L} \text { ) }\end{array}$ & $\begin{array}{l}\text { 13/54\#\# } \\
(24.1 \%)\end{array}$ & $\begin{array}{l}\text { 19/178\#\# } \\
(10.7 \%)\end{array}$ & $\begin{array}{l}32 / 232 \\
(13.8 \%)\end{array}$ & $\begin{array}{l}16 / 41 \# \\
(9 \%)\end{array}$ & $\begin{array}{l}26 / 148 \# \\
(17.6 \%)\end{array}$ & $\begin{array}{l}42 / 189 * * \\
(22.2 \%)\end{array}$ & $\begin{array}{l}12 / 29 \# \\
(41.4 \%)\end{array}$ & $\begin{array}{l}\text { 14/112\# } \\
(12.5 \%)\end{array}$ & $\begin{array}{l}26 / 141 \\
(18.4 \%)\end{array}$ & $\begin{array}{l}6 / 22 \# \# \\
(27.3 \%)\end{array}$ & $\begin{array}{l}6 / 72 \neq \\
(8.3 \%\end{array}$ \\
\hline $\begin{array}{l}\text { Severe } \\
\text { hypocalcemia (iCa } \\
<0.9 \mathrm{mmol} / \mathrm{L} \text { ) }\end{array}$ & $\begin{array}{l}4 / 54 \\
(7.4 \%)\end{array}$ & $\begin{array}{l}8 / 178 \\
(4.5 \%)\end{array}$ & $\begin{array}{l}12 / 232 \\
(5.2 \%)\end{array}$ & $\begin{array}{l}5 / 41 \\
(12.2 \%)\end{array}$ & $\begin{array}{l}8 / 148 \\
(5.4 \%)\end{array}$ & $\begin{array}{l}13 / 189 \\
(6.9 \%)\end{array}$ & $\begin{array}{l}2 / 29 \\
(6.9 \%)\end{array}$ & $\begin{array}{l}4 / 112 \\
(3.6 \%)\end{array}$ & $\begin{array}{l}6 / 141 \\
(4.2 \%)\end{array}$ & $\begin{array}{l}2 / 22 \\
(9.1 \%)\end{array}$ & $\begin{array}{l}0 / 72 \\
(0 \%)\end{array}$ \\
\hline $\begin{array}{l}\text { Hypercalcemia } \\
\text { (iCa > } \\
1.21 \mathrm{mmol} / \mathrm{L} \text { ) }\end{array}$ & $\begin{array}{l}9 / 54 \\
(16.7 \%)\end{array}$ & $\begin{array}{l}23 / 178 \\
(12.9 \%)\end{array}$ & $\begin{array}{l}32 / 232 \\
(13.8 \%)\end{array}$ & $\begin{array}{l}3 / 41 \\
(7.3 \%)\end{array}$ & $\begin{array}{l}2 / 148 \\
(1.6 \%)\end{array}$ & $\begin{array}{l}5 / 189 * \\
(2.6 \%)\end{array}$ & $\begin{array}{l}3 / 29 \\
(10.3 \%)\end{array}$ & $\begin{array}{l}9 / 112 \\
(8 \%)\end{array}$ & $\begin{array}{l}12 / 141 \\
(8.5 \%)\end{array}$ & $\begin{array}{l}0 / 22 \\
(0 \%)\end{array}$ & $\begin{array}{l}5 / 72 \\
(6.9 \%\end{array}$ \\
\hline $\begin{array}{l}\text { Severe } \\
\text { hypercalcemia } \\
\text { (iCa > } \\
1.32 \mathrm{mmol} / \mathrm{L} \text { ) }\end{array}$ & $\begin{array}{l}2 / 54 \\
(3.7 \%)\end{array}$ & $\begin{array}{l}2 / 178 \\
(1.1 \%)\end{array}$ & $\begin{array}{l}4 / 232 \\
(1.7 \%)\end{array}$ & $\begin{array}{l}0 / 41 \\
(0 \%)\end{array}$ & $\begin{array}{l}1 / 148 \\
(0.7 \%)\end{array}$ & $\begin{array}{l}1 / 189 \\
(0.5 \%)\end{array}$ & $\begin{array}{l}0 / 29 \\
(0 \%)\end{array}$ & $\begin{array}{l}1 / 112 \\
(0.9 \%)\end{array}$ & $\begin{array}{l}1 / 141 \\
(0.7 \%)\end{array}$ & $\begin{array}{l}0 / 22 \\
(0 \%)\end{array}$ & $\begin{array}{l}1 / 72 \\
(1.4 \%\end{array}$ \\
\hline $\begin{array}{l}\text { Metabolic alkalosis } \\
(\mathrm{pH}>7.45 \text { and } \\
\mathrm{HCO}_{3}^{-} \\
>26 \mathrm{mmol} / \mathrm{L})\end{array}$ & $\begin{array}{l}8 / 67 \\
(11.9 \%)\end{array}$ & $\begin{array}{l}29 / 165 \\
(17.6 \%)\end{array}$ & $\begin{array}{l}37 / 232 \\
(15.9 \%)\end{array}$ & $\begin{array}{l}11 / 53 \\
(20.7 \%)\end{array}$ & $\begin{array}{l}33 / 136 \\
(24.3 \%)\end{array}$ & $\begin{array}{l}44 / 189 \\
(23.3 \%)\end{array}$ & $\begin{array}{l}13 / 37 \\
(35.1 \%)\end{array}$ & $\begin{array}{l}26 / 104 \\
(25 \%)\end{array}$ & $\begin{array}{l}39 / 141 * \\
(27.7 \%)\end{array}$ & $\begin{array}{l}8 / 26 \\
(30.8 \%)\end{array}$ & $\begin{array}{l}16 / 6 \varepsilon \\
(23.6 ?\end{array}$ \\
\hline HAGMA & $\begin{array}{l}\text { 9/66\#\# } \\
(13.6 \%)\end{array}$ & $\begin{array}{l}9 / 165 \# \# \\
(5.5 \%)\end{array}$ & $\begin{array}{l}18 / 231 \\
(7.8 \%)\end{array}$ & $\begin{array}{l}1 / 53 \\
(1.97 \%)\end{array}$ & $\begin{array}{l}4 / 136 \\
(2.9 \%)\end{array}$ & $\begin{array}{l}5 / 189 * * \\
(2.6 \%)\end{array}$ & $\begin{array}{l}0 / 37 \\
(0 \%)\end{array}$ & $\begin{array}{l}2 / 104 \\
(1.9 \%)\end{array}$ & $\begin{array}{l}2 / 141^{*} \\
(1.4 \%)\end{array}$ & $\begin{array}{l}0 / 26 \\
(0 \%)\end{array}$ & $\begin{array}{l}0 / 68 \\
(0 \%)\end{array}$ \\
\hline $\mathrm{tCa} / \mathrm{iCa}>2,5$ & $\begin{array}{l}0 / 27 \\
(0 \%)\end{array}$ & $\begin{array}{l}2 / 89 \\
(2.2 \%)\end{array}$ & $\begin{array}{l}2 / 116 \\
(1.7 \%)\end{array}$ & $\begin{array}{l}0 / 30 \\
(0 \%)\end{array}$ & $4 / 80(5 \%)$ & $\begin{array}{l}4 / 110 \\
(3.6 \%)\end{array}$ & $\begin{array}{l}0 / 19 \\
(0 \%)\end{array}$ & $\begin{array}{l}7 / 62 \\
(11.3 \%)\end{array}$ & $\begin{array}{l}7 / 81 * * \\
(8.6 \%)\end{array}$ & $\begin{array}{l}0 / 14 \\
(0 \%)\end{array}$ & $\begin{array}{l}0 / 36 \\
(0 \%)\end{array}$ \\
\hline $\begin{array}{l}\text { Citrate } \\
\text { accumulation }\end{array}$ & $\begin{array}{l}1 / 27 \\
(3.7 \%)\end{array}$ & $\begin{array}{l}4 / 89 \\
(4.5 \%)\end{array}$ & $\begin{array}{l}5 / 116 \\
(4.3 \%)\end{array}$ & $\begin{array}{l}1 / 30 \\
(3.3 \%)\end{array}$ & $\begin{array}{l}5 / 80 \\
(6.2 \%)\end{array}$ & $\begin{array}{l}6 / 110 \\
(5.5 \%)\end{array}$ & $\begin{array}{l}0 / 19 \\
(0 \%)\end{array}$ & $\begin{array}{l}2 / 61 \\
(3.3 \%)\end{array}$ & $\begin{array}{l}2 / 80 \\
(2.5 \%)\end{array}$ & $\begin{array}{l}1 / 14 \\
(7.1 \%)\end{array}$ & $\begin{array}{l}0 / 36 \\
(0 \%)\end{array}$ \\
\hline
\end{tabular}

Post-dilution haemofiltration with the RCA protocol provided stable sodium and chloride concentrations, with their median values approaching the midreference values, and decreased ranges throughout the course of treatment (Fig. 2). 


\section{Calcium}

The incidences of hypercalcaemia $(>1.21 \mathrm{mmol} / \mathrm{L}$ ) and severe hypercalcaemia $(>1.32 \mathrm{mmol} / \mathrm{L})$ during haemofiltration were $5.2 \%$ and $0.7 \%$, respectively. The incidences of hypercalcaemia were significantly lower after 24 and 72 hours of $\mathrm{CVVH}$ than at baseline. The incidences of hypocalcaemia (<0.98 mmol/L) and severe hypocalcaemia ( $<0.9 \mathrm{mmol} / \mathrm{L})$ during haemofiltration treatment were $17.9 \%$ and $0.7 \%$, respectively. The incidence of hypocalcaemia was significantly higher after 24 hours of haemofiltration than at baseline. The incidence of hypocalcaemia was higher in survivors before the beginning and after 24,48 and 72 hours of haemofiltration. (Fig. 3)

Altogether, out of all 246 measurements at which data for the calculation of the tCa/iCa ratio were complete after the commencement of haemofiltration treatment, its value exceeded 2.5 at 11 samples (4.5\%), including two at which $3<\mathrm{tCa} / \mathrm{iCa} \leq 3.5$ and one at which it exceeded 3.5 . All episodes in which $\mathrm{tCa} / \mathrm{iCa}$ exceeded 2.5 were observed in 10 patients from the non-surviving group. The incidence of a tCa/iCa ratio higher than 2.5 was significantly higher after 48 hours of haemofiltration than at baseline, and no significant inter-group difference was observed. The incidence of citrate accumulation did not differ throughout haemofiltration treatment, and no inter-group difference was observed.

\section{Magnesium and phosphate}

Magnesium and phosphate concentrations significantly decreased during haemofiltration, but no significant difference between the outcome groups was observed (Fig. 4. A and B) Moreover, hypomagnesaemia was reported in 64/287 (20.6\%) blood samples during CVVH. The incidence of hypomagnesaemia was significantly higher after 48 hours of haemofiltration than at baseline. After 24 hours of haemofiltration, the incidence of hypomagnesaemia was higher in survivors than in non-survivors.

Hypophosphataemia was reported in 77/247 blood samples (31\%) over the course of haemofiltration treatment. The incidence of hypophosphataemia was significantly higher after 48 and 72 hours of haemofiltration than at baseline; however, the difference after 24 hours of treatment did not reach statistical significance. The incidences of hypophosphataemia after 24,48 and 72 hours of haemofiltration were significantly higher in survivors than in non-survivors. Acid-base balance

The $\mathrm{pH}$ value and $\mathrm{HCO}_{3}{ }^{-}$concentration increased significantly during haemofiltration, and the $\mathrm{pH}$ was significantly higher in survivors than in non-survivors (Fig. 5). During haemofiltration treatment, metabolic alkalosis ( $\mathrm{pH}>7.45$ and $\mathrm{HCO}_{3}{ }^{-}>26 \mathrm{mmol} / \mathrm{L}$ ) was observed in 107 of 424 blood samples (25.2\%). The incidence of metabolic alkalosis was significantly higher after 48 and 72 hours of CVVH than at baseline. Altogether, out of 637 measurements, high anion gap metabolic acidosis (HAGMA) was observed at 25 time points (3.9\%). The incidence of HAGMA during haemofiltration treatment did not differ between survivors and non-survivors, but it was significantly higher in survivors than in non-survivors before the beginning of haemofiltration. The incidence of HAGMA was significantly lower after 24,48 and 72 hours of haemofiltration than at baseline.

\section{Lactate}

Both blood lactate and anion gap changed significantly during treatment ( $p=0.001$ and $p=0.026$, respectively). However, no significant inter-group difference was observed (Fig. 6). Out of 23 sessions that started with lactate levels equal to or greater than $4 \mathrm{mmol} / \mathrm{L}$, the tCa/iCa ratio was tracked in 17 sessions. Within these sessions, the $\mathrm{tCa} / \mathrm{iCa}$ ratio exceeded 2.5 in 3 sessions, but during further $\mathrm{CVVH}$ treatment, it felt below 2.5 in one session and decreased from 2.58 to 2.52 in another session. We did not find a significant correlation between the lactate concentration before the beginning of the haemofiltration session and the tCa/iCa ratio after 24, 48 and 72 hours of haemofiltration in linear regression (Fig. 7).

\section{Citrate accumulation}

Altogether, out of all 246 time points at which data for the calculation of the tCa/iCa ratio were complete after the initiation of haemofiltration treatment, its value exceeded 2.5 at 11 time points (4.5\%), including two at which $3<\mathrm{tCa} / \mathrm{iCa} \leq 3.5$ and one at which it exceeded 3.5. All episodes in which tCa/iCa exceeded 2.5 were observed in 10 patients from the non-surviving group. The incidence of a tCa/iCa ratio higher than 2.5 was significantly higher after 48 hours of haemofiltration than at baseline, and no significant inter-group difference was observed. The incidence of CA did not differ over the course of haemofiltration treatment, and no inter-group difference was observed.

\section{Discussion}

\section{Main finding}

To the best of our knowledge, this is the first study to evaluate electrolytic homeostasis and metabolic control during the simplified ACD-A RCA protocol for $\mathrm{CVVH}$ on the Aquarius platform. In this prospective study, we found that CVVH with a simplified RCA protocol provides very good sodium and chloride balance, but it is related to significant incidences of magnesium and phosphate deficiency. We also found that the incidence of CA can be significant in cardiovascular surgery patients. Interestingly, the tCa/iCa ratio did not increase in many patients in whom CVVH was started at a high lactate level. Except for magnesium and phosphate, which should be supplemented during CVVH, the RCA protocol was found to be safe and associated with satisfactory ion and metabolic homeostasis.

\section{Ionic concentration}

When over 20 litres of body water per day is replaced with a substitution fluid, the problem of ion homeostasis must be addressed. It is possible that some ions are removed more easily than others during CRRT, and it is impossible to discuss CRRT safety without addressing the issue of ion balance. In our study, 
the serum potassium concentration was maintained within the normal range by the infusion of potassium chloride, and substitution solution bags were supplemented with potassium to reach $4 \mathrm{mEq} / \mathrm{L}$. The investigation of the incidence of potassium abnormalities was not included in the aims of the study.

\section{Sodium and chloride}

Citrate, when administered as tri-sodium, can induce hypernatraemia [18]. However, previous studies on CVVHDF with RCA also reported a significant decrease in sodium levels after treatment, which was not observed in our group [12]. The use of ACD-A solution for RCA is related to a lower sodium load than the use of tri-sodium citrate-based RCA [5]. Therefore, with the ACD-A solution-based RCA protocol, low-sodium substitution fluid is not required to prevent a patient from developing hypernatraemia. The sodium concentration in the Accusol $\mathrm{KO}$ used in this study was $140 \mathrm{mmol} / \mathrm{L}$. The trend in the sodium level towards a narrow physiologic range led to decreased incidences of both hyper- and hyponatraemia within a short time after the initiation of CVVH RCA. The $2.4 \%$ incidence of hypernatraemia observed in our group during CRRT treatment was lower than that observed with protocols based on tri-sodium citrate solutions used for CVVHD [19]. The decreased incidence of hypo- and hypernatraemia and severe hyperchloraemia during the course of haemofiltration treatment observed in our patients indicate that the CVVH RCA protocol on the Aquarius platform is close to perfect with respect to sodium and chloride balance.

\section{Calcium}

In our study, the incidence of hypocalcaemia was higher than the results reported in previous studies on RCA for continuous veno-venous haemodialysis (CVVHD) and continuous veno-venous hemodiafiltration (CVVHDF) (2.8-13\%), but this may have been due to the use of different diagnostic criteria [18, 2023]. Khadzhynov et al. observed that during RCA haemodiafiltration, up to $66 \%$ of the measured iCa concentrations were outside of the normal range, leading to a $67.5 \%$ incidence of hypocalcaemia $(<1.1 \mathrm{mmol} / \mathrm{L})$ [20]. The incidence of moderate hypocalcaemia $(<1 \mathrm{mmol} / \mathrm{L})$ reported in his study $(13.3 \%)$ was lower than that observed in our patients (18.9\%) based on similar criteria [20]. He also reported severe hypocalcaemia (<0.9 mmol/L) in three patients (20\%), while in our group, we reported severe hypocalcaemia in 11 patients (22\%), which was equal to $4.9 \%$ of all readings. Hypercalcaemia was observed more often before than during haemofiltration in the present cohort ( $4.9 \%$ of all readings during $\mathrm{CVVH}$ ), and the incidence was higher than those previously reported from CVVHD and CVVHDF studies (2-2.5\%) $[19,20]$. The incidence of severe hypercalcaemia $(0.7 \%)$ in our group was, however, lower than the $2 \%$ incidence observed by Khadzhynov [20].

The incidence of electrolyte imbalances is difficult to compare, not only due to different threshold values adopted for their identification but also due to the variance in reference values between laboratories and methods of analysis. According to our hospital laboratory, the normal range of iCa is from 0.98 to 1.21 . A change in the calcium chloride concentration used for supplementation, which took place in our study protocol, should not have significantly influenced the results, as with initially lower concentrations, higher calcium chloride solution flows were used. Although the authors increased the target calcium range from the original RCA protocol, a substantial number of iCa readings were below the normal range, which indicates that the original protocol for the adjustment of calcium substitution was inadequate [14]. This might be of importance in cardiac surgery patients, in whom low iCa can exacerbate heart failure.

\section{Magnesium}

In addition to calcium, citrate chelates magnesium ions and moves them to the filtrate. This can lead to hypomagnesaemia if magnesium replenishment with the substitution fluid is not adequate. It was observed that post-filter magnesium concentrations decrease in a manner similar to the concentrations of calcium ions under RCA [24]. When using substitution fluid containing $0.5 \mathrm{mmol} / \mathrm{L}$ magnesium, a tendency towards a decreased magnesium concentration was observed during haemofiltration treatment. Magnesium is a very important intracellular ion that is responsible for crucial physiological functions. Prolonged CRRT with RCA can cause the depletion of its limited pool of approximately 1000 mmols. Despite routine magnesium sulfate supplementation at a rate of $0.81 \mathrm{mmol} /$ hour, in our group, hypomagnesaemia was more common (22.3\%) than previously reported from the CVVHD RCA study in cancer patients with AKI (2.3\%) [19]. It was proposed that the magnesium concentration in substitution fluids used in ICU patients should be supra-normal rather than subnormal to compensate for increased losses due to its chelation by citrate [25]. Previously, supra-normal values of magnesium were observed in $54 \%$ of samples during haemodiafiltration treatment, which might increase the risk of muscle weakness [12]. In the studied patients, the magnesium level in the serum decreased over time during treatment with CVVH with A-CDA RCA. Significant hypomagnesaemia developed after 48 hours of CVVH.

\section{Inorganic phosphate}

The incidence of hypophosphataemia in ICU patients treated with CRRT without replacement solutions containing phosphate can reach up to $80 \%$ and is related to increased mortality [26]. The 34.4\% incidence of hypophosphataemia in our study is comparable to other reported results when no-phosphatecontaining substitution fluids were administered, but it is much higher than the $3 \%$ incidence reported in the study on CVVHDF with phosphate-containing fluids [27]. The observed increase in the incidence of hypophosphataemia over the course of CVVH treatment, although non-significant, could have deleterious effects on patient outcomes and should be corrected in future work to determine an optimal CVVH RCA protocol based on a phosphate-containing solution. The higher incidence of hypophosphataemia in survivors than in non-survivors might be attributed to their better general condition and lower need for parenteral nutrition. Phosphate was routinely intravenously supplemented in patients with parenteral nutrition, while it was administered only sporadically in patients who were fed enterally. As hyperphosphataemia mostly results from progressive renal dysfunction in AKI patients and could not be attributed to the CRRT protocol, the incidence of hyperphosphataemia was not assessed in this trial. The reported protocol might be improved by starting with higher supplementation doses of magnesium and inorganic phosphate than those used in the authors' department.

\section{$\mathrm{pH}$ and bicarbonate}

During CRRT with RCA, acid-base status can be affected by excess citrate, leading to metabolic alkalosis or impaired clearance, which can result in an exacerbation of metabolic acidosis. Additionally, excess chloride could contribute to metabolic acidosis. The safe implementation of RCA requires a properly designed and flexible protocol with adjustable dosing. Strict adherence to such protocols should prevent metabolic impairment. The ACD-A solution used for RCA in our study has some advantages over the most commonly used tri-sodium citrate solution. Its use is related to the generation of $1 / 3$ less bicarbonate after metabolism. A target citrate concentration in the filter compartment equal to $2.8 \mathrm{mmol} / \mathrm{L}$ should decrease the iCa concentration to $0.35 \mathrm{mmol} / \mathrm{L}$ on

Page $8 / 26$ 
average [14] and provide effective anticoagulation [16]. According to the protocol, citrate flow was reduced stepwise when a trend towards alkalosis was observed, although this did not sufficiently counteract the development of metabolic alkalosis. The episodes of metabolic acidosis were most common at the beginning of CRRT therapy.

In the present study, the $\mathrm{pH}$ value and bicarbonate concentration increased significantly over time. Metabolic alkalosis during CRRT was more common in our study (25.2\%) than in earlier studies on RCA for CVVHD: $14 \%$ observed in cancer patients [19] and 5\% observed in ICU patients [20, 28]. However, this overcompensation of metabolic acidosis with the Aquarius CVVH RCA protocol might be beneficial in patients who are acidotic at the beginning of CRRT. A trend towards a higher $\mathrm{pH}$ and a higher bicarbonate concentration may indicate the presence of $\mathrm{CA}$. It is a situation in which the capacity for citrate metabolism is not saturated and all citrate-calcium complexes are metabolized. The resulting net load of sodium leads to plasma alkalization by the increased strong ion difference, but an increase in the $\mathrm{tCa} / \mathrm{iCa}$ ratio is not observed, and the iCa level remains normal.

In a previous study on CVVH with isosmotic citrate anticoagulation, Cassina et al. reported alkalosis ( $\mathrm{pH}>7.48)$ mostly of the respiratory type in only $4 \%$ of patients [11]. In contrast to our results, Khadzhynov et al. found that the bicarbonate concentration and base excess were below the normal ranges (69.9 and $84.6 \%$, respectively) during RCA haemodiafiltration [12]. As also reported in previous studies, the results of our study indicated that most specific RCA protocols can cause upward or downward trends in bicarbonate concentrations and base excess. Jacobs et al. reported metabolic alkalosis ( $\mathrm{pH}>7.5$ ) in $10 \%$ of patients treated with pre-dilution CVVH RCA in the group of patients treated with Prismocitrate 18 solution in contrast to its absence in patients treated with Prismocitrate 10/2 solution [29].

\section{High anion gap metabolic acidosis (HAGMA)}

HAGMA is frequently observed in conjunction with CA [5]. HAGMA and increased lactate concentrations are believed to appear not secondary to CA itself but rather to the shared primary problem of an impaired Krebs cycle, which reduces both citrate and lactate metabolism [5]. In our cohort, HAGMA was observed at less than $1.6 \%$ of all time points during haemofiltration, and it was lower during haemofiltration than at baseline. The low incidence reported in this study, despite the higher incidence of high anion gap, might result from trends towards a higher $\mathrm{pH}$ and a higher bicarbonate concentration.

\section{Lactate}

In our study, 23 haemofiltration sessions (10\%) were started at a lactate concentration $4 \mathrm{mmol} / \mathrm{L}$ or above. However, it was observed that the risk of CA during RCA is low even in cases of severe hyperlactataemia and that lactate kinetics rather than concentrations should be considered in the assessment of the risk of CA [20]. In contrast to Tan et al., who found that hyperlactataemia predicted citrate intolerance, we did not find a correlation between lactate concentration before the initiation of RCA and the tCa/iCa ratio during haemofiltration treatment (Fig. 6) [13]. We postulate that rather than a single high lactate concentration, its increasing trend or even predicted lactate clearance after the initiation of CRRT should be considered when weighing the advantages and disadvantages of RCA in critical care patients.

\section{Citrate accumulation}

It has been suggested that each new CRRT RCA protocol should be assessed with respect to the incidence of CA and electrolytic disorders to enable clinicians to predict and possibly prevent potentially dangerous shifts and complications [12]. The measurement of the citrate concentration in the plasma is still not feasible or timely [22]. An increased tCa/iCa ratio is a reliable indicator of CA [5]. A tCa/iCa threshold of 2.5 is commonly used as an indication of CA; however, it has high specificity but low sensitivity as a risk factor for mortality $[13,30]$. Some authors have proposed that this threshold should be decreased to 2.3 , while others claim that it may be a poor indicator of ongoing accumulation [5, 30, 31].

Strategies have been developed to prevent CA. The citrate infusion rate can be significantly decreased from the initial dose. This must be accompanied by a decrease in the blood flow and the replacement fluid flow, rendering CRRT less effective, however theoretically without increasing the risk of filter clotting. An additional option is to continue up-titrating the calcium chloride infusion. Finally, if the ratio continues to increase, citrate anticoagulation can be replaced with systemic anticoagulation, or CRRT can be continued without anticoagulation [15].

Impaired citrate metabolism causes the build-up of calcium-citrate complexes, resulting in impaired free calcium recuperation. In fact, low iCa is the only known effect of citrate toxicity. Clinical symptoms in humans appear when the $\mathrm{Ca}^{++}$concentration falls below $0.8 \mathrm{mmol} / \mathrm{L}$. The first symptom is usually a prolonged QTc interval. With a further decrease in $\mathrm{Ca}^{++}$levels, patients present with decreased cardiac contractility, hypotension, tremor, muscle weakness, coagulopathy, nausea and vomiting, and cardiac arrest [32]. In animal studies, the maximum citrate infusion rate without clinical symptoms was $0.67-$ $1.00 \mathrm{mmol} / \mathrm{kg} /$ hour. In our study, the case of low iCa was observed in a patient with severe lactic acidosis, who should be excluded from citrate anticoagulation. In the studied patients, other indirect features of CA, such as escalating calcium requirements and the development of HAGMA, were rarely observed, making the diagnosis of citrate toxicity questionable. In the present study, the incidence of a high tCa/iCa ratio during haemofiltration treatment (4.6\%) was higher than that observed during RCA for CVVHD, when it did not exceed 2.3 in any patient [19].

It is still a matter of debate whether RCA can be used in patients with high lactate levels before the initiation of CRRT. In our group, the median value of the lactate concentration did not increase, indicating that citrate anticoagulation did not inhibit lactate clearance, presumably due to adequate tissue perfusion in most of the patients. In our group, the $3.8 \%$ incidence of CA was slightly higher than the $2.3 \%$ reported by Khadzhynov et al. in a study with general ICU patients treated with CVVHD. However, in his study, patients with CA after over 48 hours of CRRT were excluded, so the entire incidence of CA was 3.8\% [20]. The authors of this study concluded that the risk of CA during RCA is low even in cases of initial severe hyperlactataemia. During CVVH with RCA, the incidence of CA may be as high as $8-23 \%$, depending on the CRRT dose $[13,33]$. It should be emphasized that in our group, at three measurements, the $\mathrm{tCa} / \mathrm{iCa}$ ratio was higher than 3.0, indicating a high risk of citrate toxicity. A tCa/iCa ratio up to 3.4 was previously reported in studies on RCA with high-dose haemofiltration (35-45 ml/kg/h) [13]. In our group, a higher incidence of CA was expected as a result of higher blood flows during CVVH, which require higher citrate doses than in most CVVHD protocols. Tan et al. observed citrate intolerance in 22.7\% of patients treated with RCA haemofiltration [13]. Data from the 
literature on the incidence of CA in patients with hyperlactataemia treated with RCA CRRT are limited, and questions arise regarding whether refusing these patients the benefits of RCA is reasonable [20]. Our study is not exceptional in the fact that RCA CRRT was started in patients with hyperlactataemia. In a previously described group of 1049 patients, CRRT was started in 221 ICU patients with lactate levels exceeding 4 mmol/L, in whom the reported incidence of CA was $6.3 \%[20]$.

\section{Strengths And Limitations}

The strengths of our study include the reliance on the prospective evaluation of a considerable number of CVVH procedures in cardiovascular surgery patients using a standard RCA protocol. To the best of the authors' knowledge, this is the first study to describe acid-base imbalance and electrolytic disorders during a simplified CVVH RCA protocol without the need for post-filter calcium assessment. Our results indicate that the evaluated protocol can be safely used in cardiovascular surgery patients, provided that magnesium and phosphate removal is adequately and aggressively supplemented. Our study evaluated acidbase imbalance and electrolyte disorders with different blood flow settings and CRRT doses, thus representing real-life everyday clinical practice.

Our study shares the limitations of all single-centre studies. An additional limitation was that the analysis was not restricted to only the first CVVH session per patient, so subsequently analysed sessions for the same patient may have serious confounding factors and were not truly independent. The time frames for the interruptions in CVVH treatment varied from 2 hours to several days, depending on the clinical situation and indication to reinstitute CRRT. This type of study protocol could potentially result in bias in the electrolyte and acid-base analyses; for example, in some patients, the symptoms of CA were observed before beginning haemofiltration. This flaw in the study protocol, however, should not decrease but rather increase the incidence of electrolyte and acid-base imbalances, decreasing the risk of the underestimation of CA and other abnormalities. Another drawback of this study was that in a substantial number of patients, data on magnesium, phosphate and tCa were not available, which limited the assessment of certain imbalances to subgroups of patients.

\section{Conclusions}

The present study showed that the simplified RCA protocol for CVVH on the Aquarius platform in cardiovascular surgery patients provides excellent sodium and chloride balance. Losses of magnesium and phosphate during CVVH therapy can lead to the depletion of these ions. The simplified RCA protocol provided insufficient control of the acid-base balance, causing over-compensation for metabolic acidosis and leading to metabolic alkalosis. The evaluated protocol might be related to significant incidences of hypocalcaemia and CA. Whether these are higher than those associated with other RCA protocols should be clarified in a comparative study.

\section{List Of Abbreviations}

ACD-A - acid dextrose formula A;

AKI - acute kidney injury;

ASA - acetylsalicylic acid (ASA);

BUN - blood urea nitrogen;

CA - citrate accumulation;

CABG - coronary artery bypass graft;

COPD - chronic obstructive pulmonary disease;

CRRT - continuous renal replacement therapy;

CVVHD - continuous veno-venous haemodialysis;

CVVHDF - continuous veno-venous hemodiafiltration;

CVVHF - continuous veno-venous haemofiltration;

ECMO - extracorporeal membrane oxygenation;

ESRF - end-stage renal failure;

HAGMA - high anion gap metabolic acidosis;

iCa - ionized calcium (in blood);

INR - international normalized ratio;

IQR - interquartile range;

LMWH - low molecular weight heparin; 
OPCABG - off-pump coronary artery bypass graft;

PM - pacemaker;

RCA - regional citrate anticoagulation;

tCa - total calcium (in blood);

tCa/iCa - total to ionized calcium ratio;

VAD - ventricular assist device.

\section{Declarations}

Ethics approval and consent to participate: The study protocol conformed to the Ethical Principles for Medical Research Involving Human Subjects outlined in the Declaration of Helsinki, was approved by the local independent ethics committee for scientific research (NKBBN/539/2016-17) and was retrospectively registered in the ClinicalTrials database (NCT03836742). Due to the observational nature of the study, the institutional review board waived the need to obtain patient consent.

Consent for publication: not applicable.

Availability of data: The data underlying this article are available in the ZENODO repository [34].

Competing interests: RL declares receiving lecture grants from Nikkiso Poland Sp. z o.o and Fresenius Medical Care Polska S.A.

Funding: none.

Authors contribution: AK had the initial conception of the study, collected and analysed the vast parts of data, drafted partially the methods, results and discussion; MMK collected partially the data, drafted and critically reviewed the manuscript and drawed the graphical abstract; ALM collected and analyzed the data, drafted partially the methods, results, and discussion; WK and DJ took part in study design, reviewed the analyses and drafted partially the introduction, results and discussion; RL developed the conception and designed the study, performed the analyses and prepared the figures, drafted partially the introduction, results and discussion. All authors added substantially to the intellectual content, have revised and finally approved the submitted version.

Acknowledgments: none

\section{References}

1. Pickering JW, James MT, Palmer SC: Acute kidney injury and prognosis after cardiopulmonary bypass: a meta-analysis of cohort studies. Am J Kidney Dis 2015, 65(2):283-293.

2. Kellum JAKAWG: KDIGO Clinical Practice Guideline for Acute Kidney Injury. Kidney International Supplements 2012, 2(2):1-141.

3. Stucker F, Ponte B, Tataw J, Martin PY, Wozniak H, Pugin J, Saudan P: Efficacy and safety of citrate-based anticoagulation compared to heparin in patients with acute kidney injury requiring continuous renal replacement therapy: a randomized controlled trial. Crit Care 2015, 19 (1):91.

4. Schilder L, Nurmohamed SA, ter Wee PM, Paauw NJ, Girbes AR, Beishuizen A, Beelen RH, Groeneveld AB: Citrate confers less filter-induced complement activation and neutrophil degranulation than heparin when used for anticoagulation during continuous venovenous haemofiltration in critically ill patients. BMC Nephrol 2014, 15:19.

5. Schneider AG, Journois D, Rimmelé T: Complications of regional citrate anticoagulation: accumulation or overload? Crit Care 2017, 21 (1):281.

6. Sanaie S, Mahmoodpoor A, Hamishehkar H, Shadvar K, Salimi N, Montazer M, Iranpour A, Faramarzi E: Association Between Disease Severity and Calcium Concentration in Critically III Patients Admitted to Intensive Care Unit. Anesth Pain Med 2018, 8(1):e57583.

7. Monchi M: Citrate pathophysiology and metabolism. Transfus Apher Sci 2017, 56(1):28-30.

8. Honore PM, Jacobs R, Hendrickx I, De Waele E, Van Gorp V, Spapen HD: Optimizing citrate dose for regional anticoagulation in continuous renal replacement therapy: measuring citrate concentrations instead of ionized calcium? Crit Care 2015, 19:386.

9. Upala S, Jaruvongvanich V, Wijarnpreecha K, Sanguankeo A: Hypomagnesemia and mortality in patients admitted to intensive care unit: a systematic review and meta-analysis. Qjm 2016, 109(7):453-459.

10. Wang L, Xiao C, Chen L, Zhang X, Kou Q: Impact of hypophosphatemia on outcome of patients in intensive care unit: a retrospective cohort study. BMC Anesthesiol 2019, 19(1):86.

11. Cassina T, Mauri R, Engeler A, Giannini O: Continuous veno-venous hemofiltration with regional citrate anticoagulation: a four-year single-center experience. Int J Artif Organs 2008, 31(11):937-943.

12. Khadzhynov D, Slowinski T, Lieker I, Neumayer HH, Peters H: Evaluation of acid-base control, electrolyte balance, and filter patency of a Prismaflex-based regional citrate anticoagulation protocol for pre-dilution continuous veno-venous hemodiafiltration. Clin Nephro/ 2014, 81(5):320-330.

13. Tan JN, Haroon SWP, Mukhopadhyay A, Lau T, Murali TM, Phua J, Tan ZY, Lee N, Chua HR: Hyperlactatemia Predicts Citrate Intolerance With Regional Citrate Anticoagulation During Continuous Renal Replacement Therapy. J Intensive Care Med 2019, 34(5):418-425.

Page $11 / 26$ 
14. Kirwan CJ, Hutchison R, Ghabina S, Schwarze S, Beane A, Ramsay S, Thompson E, Prowle JR: Implementation of a Simplified Regional Citrate Anticoagulation Protocol for Post-Dilution Continuous Hemofiltration Using a Bicarbonate Buffered, Calcium Containing Replacement Solution. Blood Purif 2016, 42(4):349-355.

15. Oudemans-van Straaten HM, Ostermann M: Bench-to-bedside review: Citrate for continuous renal replacement therapy, from science to practice. Crit Care 2012, 16(6):249

16. Kośka A, Kirwan CJ, Kowalik MM, Lango-Maziarz A, Szymanowicz W, Jagielak D, Lango R: Filter life span in postoperative cardiovascular surgery patients requiring continuous renal replacement therapy, using a post dilution regional citrate anticoagulation continuous hemofiltration circuit. Cardiol $J 2020$. [Epub ahead of print]

17. Zheng Y, Zhuang F, Zhu Q, Ma S, Xu Y, Lu J, Hao G, Gu Y, Hao C, Zhu M et al: Albumin-corrected total/ionized calcium ratio is not superior to total/ionized calcium ratio as an indicator of citrate accumulation. Int J Artif Organs 2017, 40(11):602-606.

18. Oudemans-van Straaten HM, Bosman RJ, Koopmans M, van der Voort PH, Wester JP, van der Spoel JI, Dijksman LM, Zandstra DF: Citrate anticoagulation for continuous venovenous hemofiltration. Crit Care Med 2009, 37(2):545-552.

19. Costa ESVT, Caires RA, Bezerra JS, Costalonga EC, Oliveira APL, Oliveira Coelho F, Fukushima JT, Soares CM, Oikawa L, Hajjar LA et al: Use of regional citrate anticoagulation for continuous venovenous hemodialysis in critically ill cancer patients with acute kidney injury. J Crit Care 2018, 47:302-309.

20. Khadzhynov D, Dahlinger A, Schelter C, Peters H, Kindgen-Milles D, Budde K, Lehner LJ, Halleck F, Staeck O, Slowinski T: Hyperlactatemia, Lactate Kinetics and Prediction of Citrate Accumulation in Critically III Patients Undergoing Continuous Renal Replacement Therapy With Regional Citrate Anticoagulation Crit Care Med 2017, 45(9):e941-e946.

21. Durão MS, Monte JC, Batista MC, Oliveira M, lizuka IJ, Santos BF, Pereira VG, Cendoroglo M, Santos OF: The use of regional citrate anticoagulation for continuous venovenous hemodiafiltration in acute kidney injury. Crit Care Med 2008, 36(11):3024-3029.

22. Monchi M, Berghmans D, Ledoux D, Canivet JL, Dubois B, Damas P: Citrate vs. heparin for anticoagulation in continuous venovenous hemofiltration: a prospective randomized study. Intensive Care Med 2004, 30(2):260-265.

23. Hetzel GR, Schmitz M, Wissing H, Ries W, Schott G, Heering PJ, Isgro F, Kribben A, Himmele R, Grabensee B et al: Regional citrate versus systemic heparin for anticoagulation in critically ill patients on continuous venovenous haemofiltration: a prospective randomized multicentre trial. Nephrol Dial Transplant 2011, 26(1):232-239.

24. Zakharchenko M, Leden P, Rulíšek J, Los F, Brodska H, Balik M: lonized Magnesium and Regional Citrate Anticoagulation for Continuous Renal Replacement Therapy. Blood Purif 2016, 41(1-3):41-47.

25. Zakharchenko M, Los F, Brodska H, Balik M: The Effects of High Level Magnesium Dialysis/Substitution Fluid on Magnesium Homeostasis under Regional Citrate Anticoagulation in Critically III. PLoS One 2016, 11(7):e0158179.

26. Pistolesi V, Zeppilli L, Polistena F, Sacco MI, Pierucci A, Tritapepe L, Regolisti G, Fiaccadori E, Morabito S: Preventing Continuous Renal Replacement Therapy-Induced Hypophosphatemia: An Extended Clinical Experience with a Phosphate-Containing Solution in the Setting of Regional Citrate Anticoagulation. Blood Purif 2017, 44(1):8-15.

27. Yang Y, Zhang P, Cui Y, Lang X, Yuan J, Jiang H, Lei W, Lv R, Zhu Y, Lai E et al: Hypophosphatemia during continuous veno-venous hemofiltration is associated with mortality in critically ill patients with acute kidney injury. Crit Care 2013, 17(5):R205.

28. Borg R, Ugboma D, Walker DM, Partridge R: Evaluating the safety and efficacy of regional citrate compared to systemic heparin as anticoagulation for continuous renal replacement therapy in critically ill patients: A service evaluation following a change in practice. J Intensive Care Soc 2017, 18(3):184192.

29. Jacobs R, Honore PM, Diltoer M, Spapen HD: Chloride content of solutions used for regional citrate anticoagulation might be responsible for blunting correction of metabolic acidosis during continuous veno-venous hemofiltration. BMC Nephro/ 2016, 17(1):119.

30. Link A, Klingele M, Speer T, Rbah R, Pöss J, Lerner-Gräber A, Fliser D, Böhm M: Total-to-ionized calcium ratio predicts mortality in continuous renal replacement therapy with citrate anticoagulation in critically ill patients. Crit Care 2012, 16(3):R97.

31. Bakker AJ, Boerma EC, Keidel H, Kingma P, van der Voort PH: Detection of citrate overdose in critically ill patients on citrate-anticoagulated venovenous haemofiltration: use of ionised and total/ionised calcium. Clin Chem Lab Med 2006, 44(8):962-966.

32. Khadzhynov D, Schelter C, Lieker I, Mika A, Staeck O, Neumayer HH, Peters H, Slowinski T: Incidence and outcome of metabolic disarrangements consistent with citrate accumulation in critically ill patients undergoing continuous venovenous hemodialysis with regional citrate anticoagulation. $J$ Crit Care 2014, 29(2):265-271.

33. Schilder L, Nurmohamed SA, Bosch FH, Purmer IM, den Boer SS, Kleppe CG, Vervloet MG, Beishuizen A, Girbes AR, Ter Wee PM et al: Citrate anticoagulation versus systemic heparinisation in continuous venovenous hemofiltration in critically ill patients with acute kidney injury: a multi-center randomized clinical trial. Crit Care 2014, 18(4):472.

34. Kośka A KMML-MA, Karolak W, Jagielak Da, Lango R: lonic Homeostasis, Acid-Base Balance and the Risk of Citrate Accumulation in Patients After Cardiovascular Surgery Treated with Continuous Veno-venous Haemofiltration with Post-Dilution Regional Citrate Anticoagulation. (Version ver. 1) [Data set]. in. Zenodo; 2020. http://doi.org/10.5281/zenodo.4073369

\section{Figures}


Flow diagram

\begin{tabular}{|c|c|c|}
\hline \multicolumn{2}{|c|}{$\begin{array}{l}\text { Included into data collection } \\
n=54 \text { patients }\end{array}$} & Enrollment \\
\hline & \multicolumn{2}{|c|}{$\begin{array}{l}\text { Excluded due to loose } \\
\text { of hemofiltration settings } \\
\text { and laboratory data }(n=2)\end{array}$} \\
\hline \multicolumn{3}{|c|}{ Included into further analysis $(n=52)$} \\
\hline & \multicolumn{2}{|c|}{$\begin{array}{l}\text { Excluded from the analysis } \\
\text { because of patients death } \\
\text { before } 24 \text { hours of CWV }(n=2)\end{array}$} \\
\hline Analysed $(n=50$ & & Analysis \\
\hline
\end{tabular}

\section{Figure 1}

Flow chart of the study population 
Flow diagram

\begin{tabular}{|c|c|c|}
\hline \multicolumn{2}{|c|}{$\begin{array}{l}\text { Included into data collection } \\
n=54 \text { patients }\end{array}$} & Enrollment \\
\hline & \multicolumn{2}{|c|}{$\begin{array}{l}\text { Excluded due to loose } \\
\text { of hemofiltration settings } \\
\text { and laboratory data }(n=2)\end{array}$} \\
\hline \multicolumn{3}{|c|}{ Included into further analysis $(n=52)$} \\
\hline & \multicolumn{2}{|c|}{$\begin{array}{l}\text { Excluded from the analysis } \\
\text { because of patients death } \\
\text { before } 24 \text { hours of CWV }(n=2)\end{array}$} \\
\hline Analysed $(n=50$ & & Analysis \\
\hline
\end{tabular}

\section{Figure 1}

Flow chart of the study population 

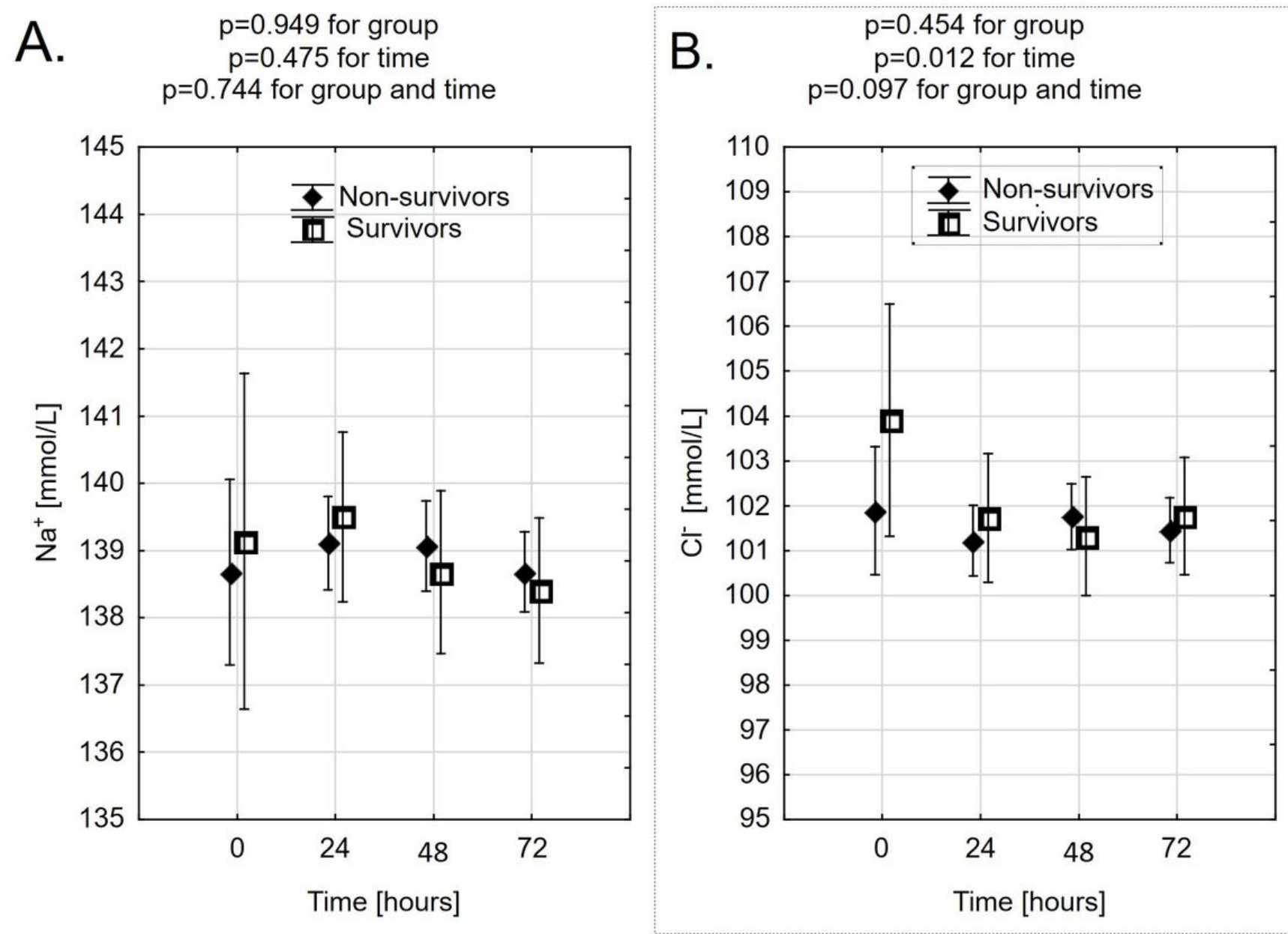

Figure 2

Sodium (A) and chloride (B) concentrations during haemofiltration treatment in survivors $(n=17)$ and non-survivors $(n=33)$ in sessions lasting $>72$ hours. (means $\pm S D$ ) 

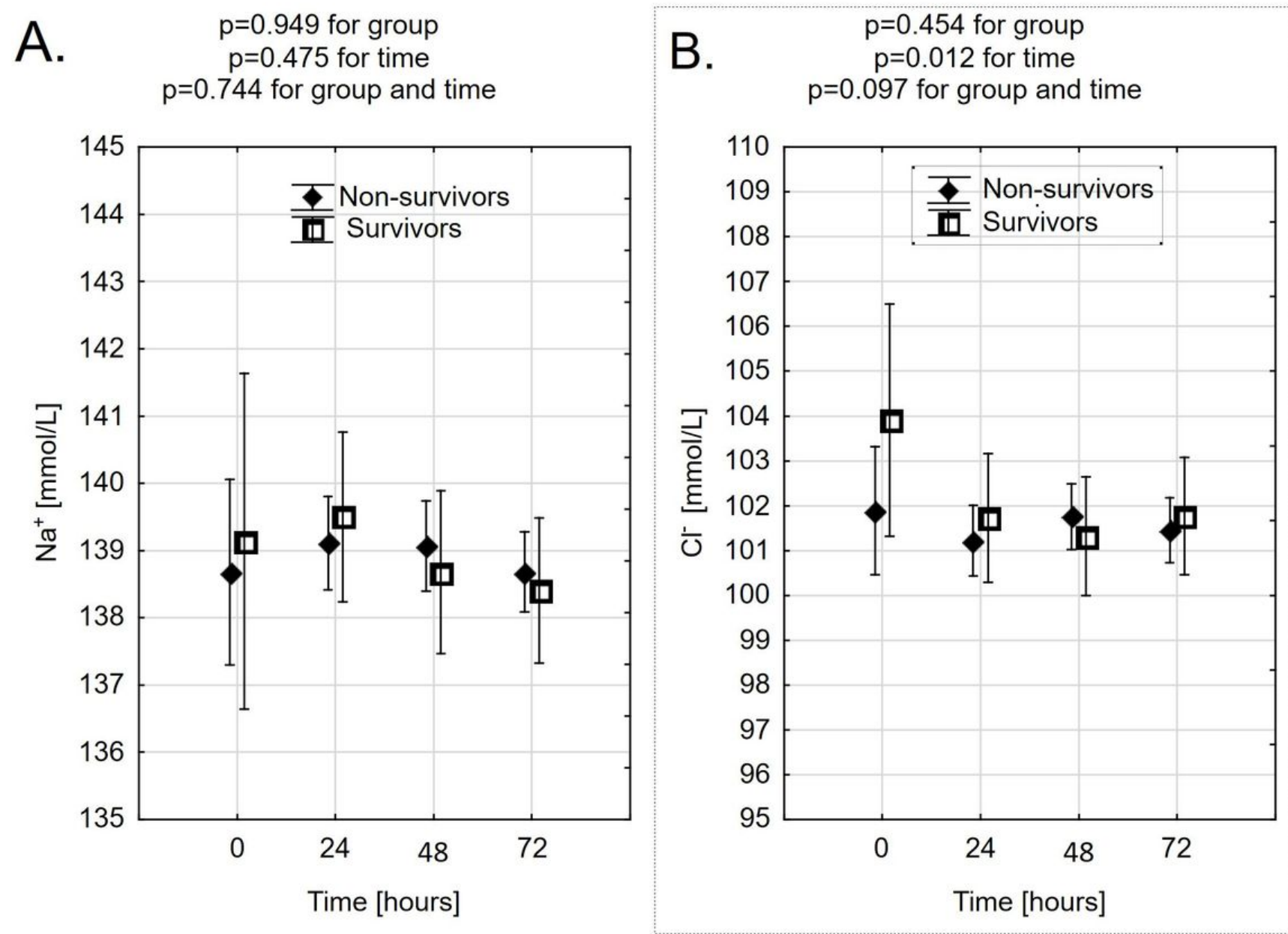

Figure 2

Sodium (A) and chloride (B) concentrations during haemofiltration treatment in survivors $(n=17)$ and non-survivors $(n=33)$ in sessions lasting $>72$ hours. (means $\pm S D$ ) 


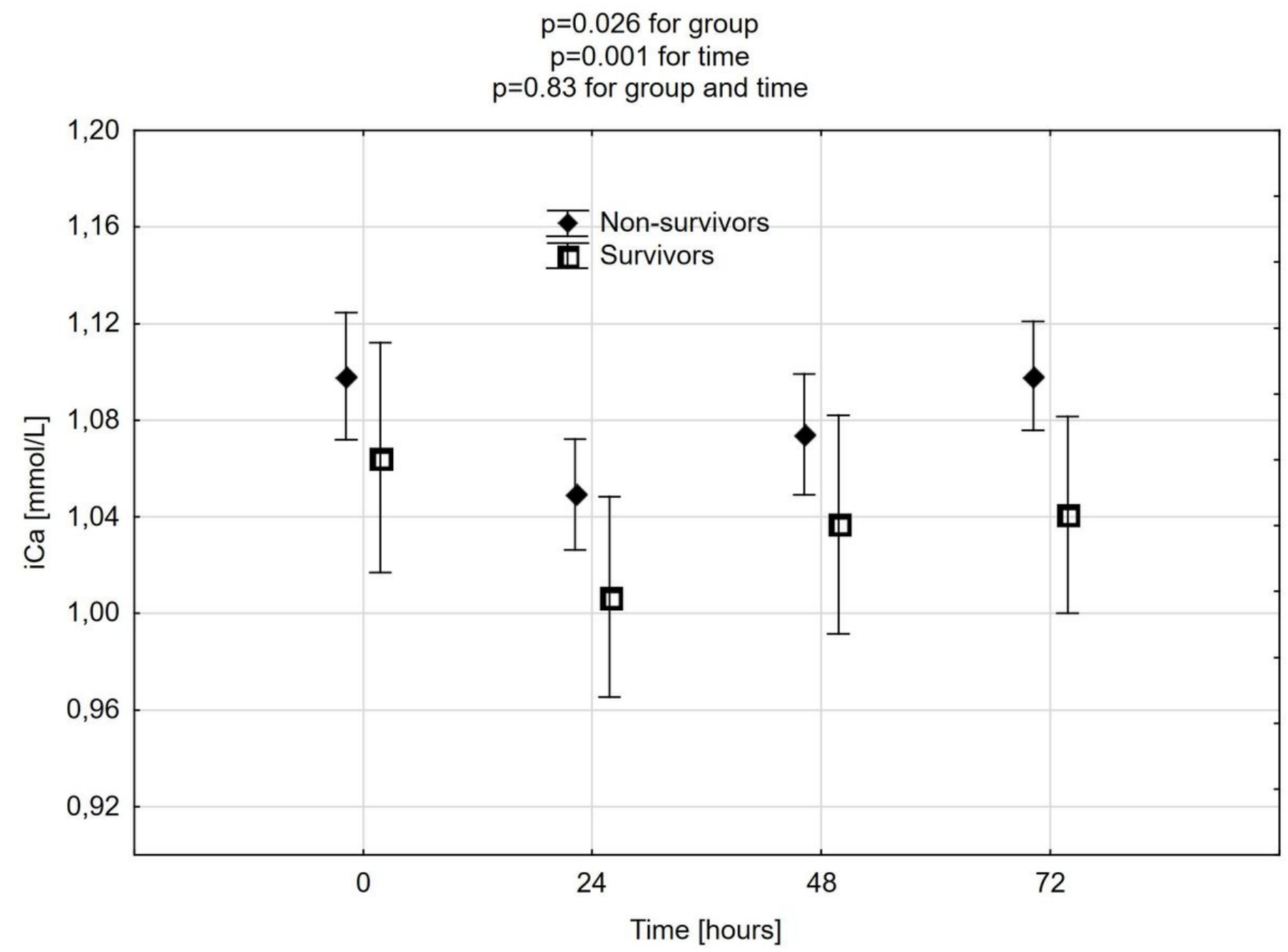

Figure 3

Ionized calcium concentration during haemofiltration treatment in survivors $(n=17)$ and non-survivors $(n=33)$ in sessions lasting $>72$ hours. $($ means \pm SD) 


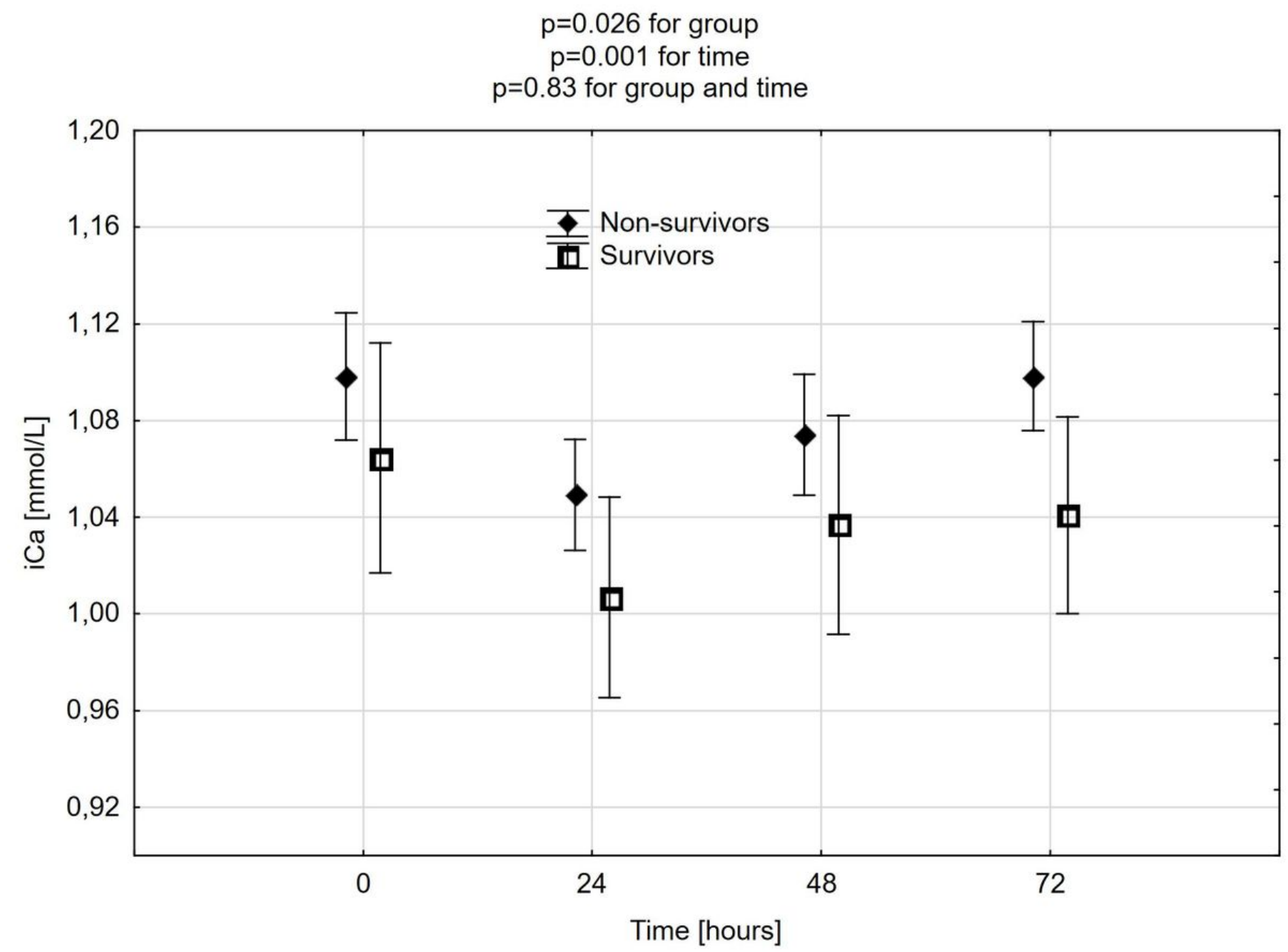

Figure 3

Ionized calcium concentration during haemofiltration treatment in survivors $(n=17)$ and non-survivors $(n=33)$ in sessions lasting $>72$ hours. $($ means \pm SD) 

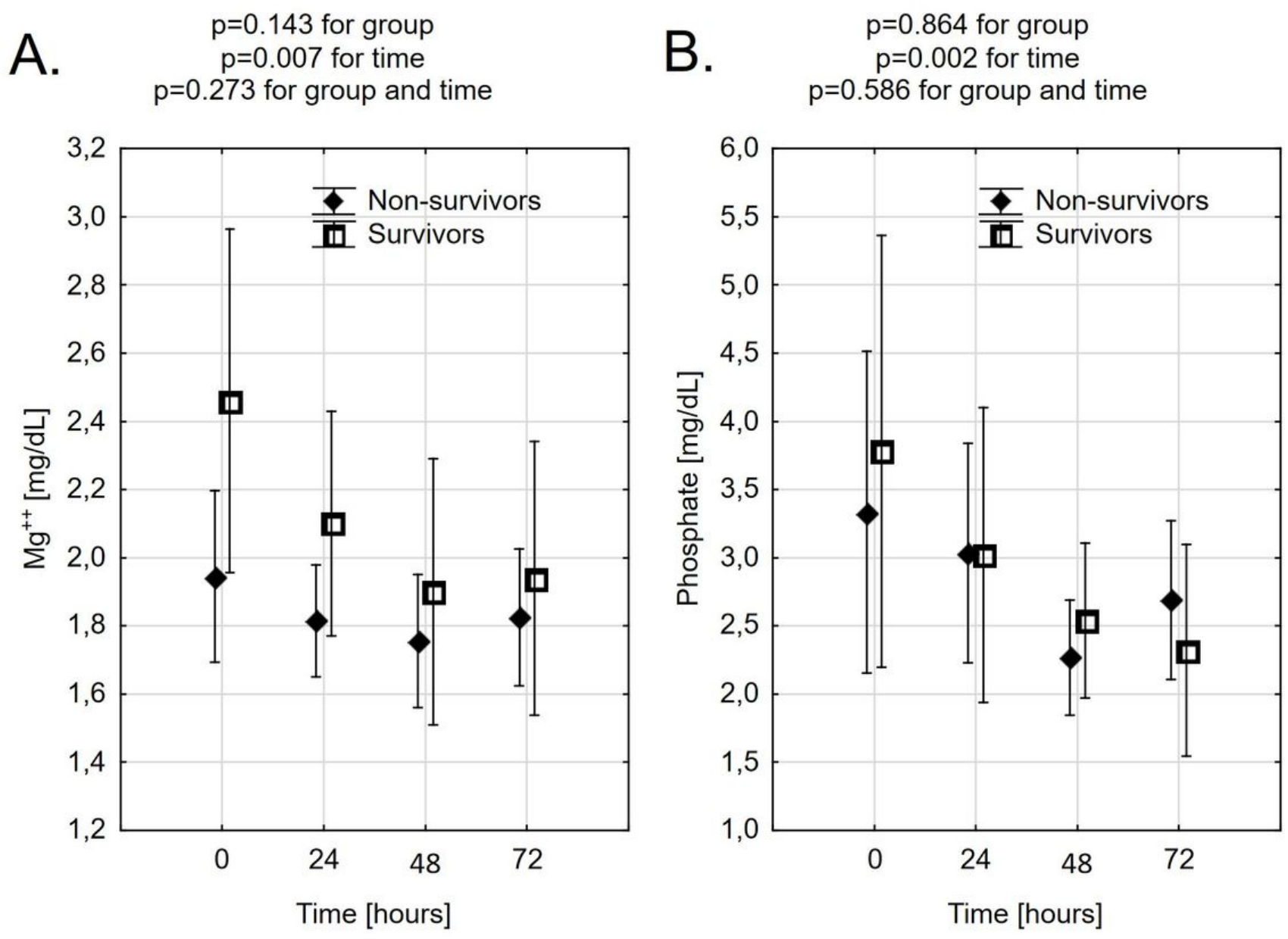

Figure 4

Magnesium (A) and phosphate (B) concentrations during sessions lasting longer than 72 hours in survivors and non-survivors. Complete data on magnesium and phosphate concentrations were available from 25 and 15 haemofiltration sessions, respectively. 

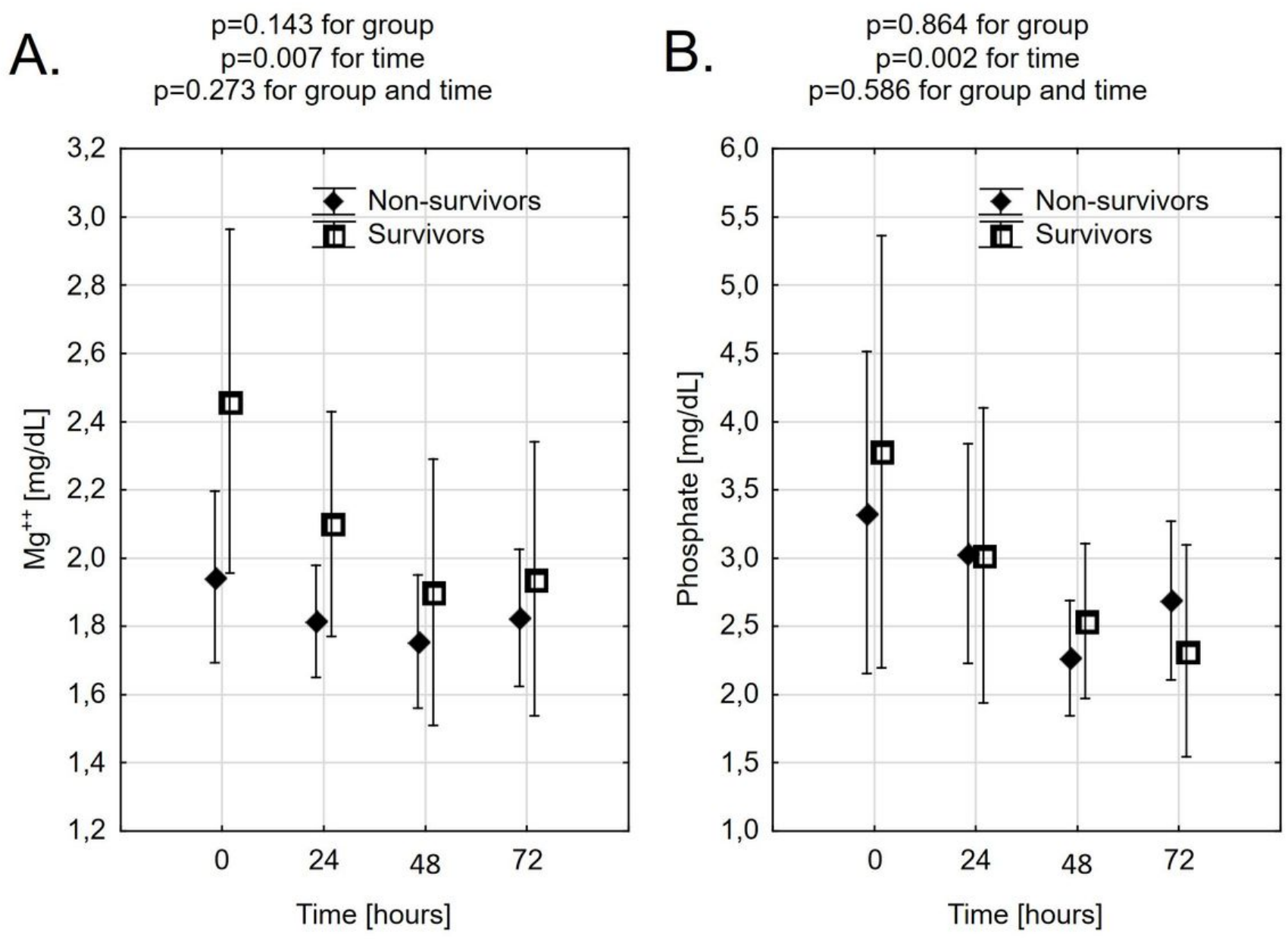

Figure 4

Magnesium (A) and phosphate (B) concentrations during sessions lasting longer than 72 hours in survivors and non-survivors. Complete data on magnesium and phosphate concentrations were available from 25 and 15 haemofiltration sessions, respectively. 

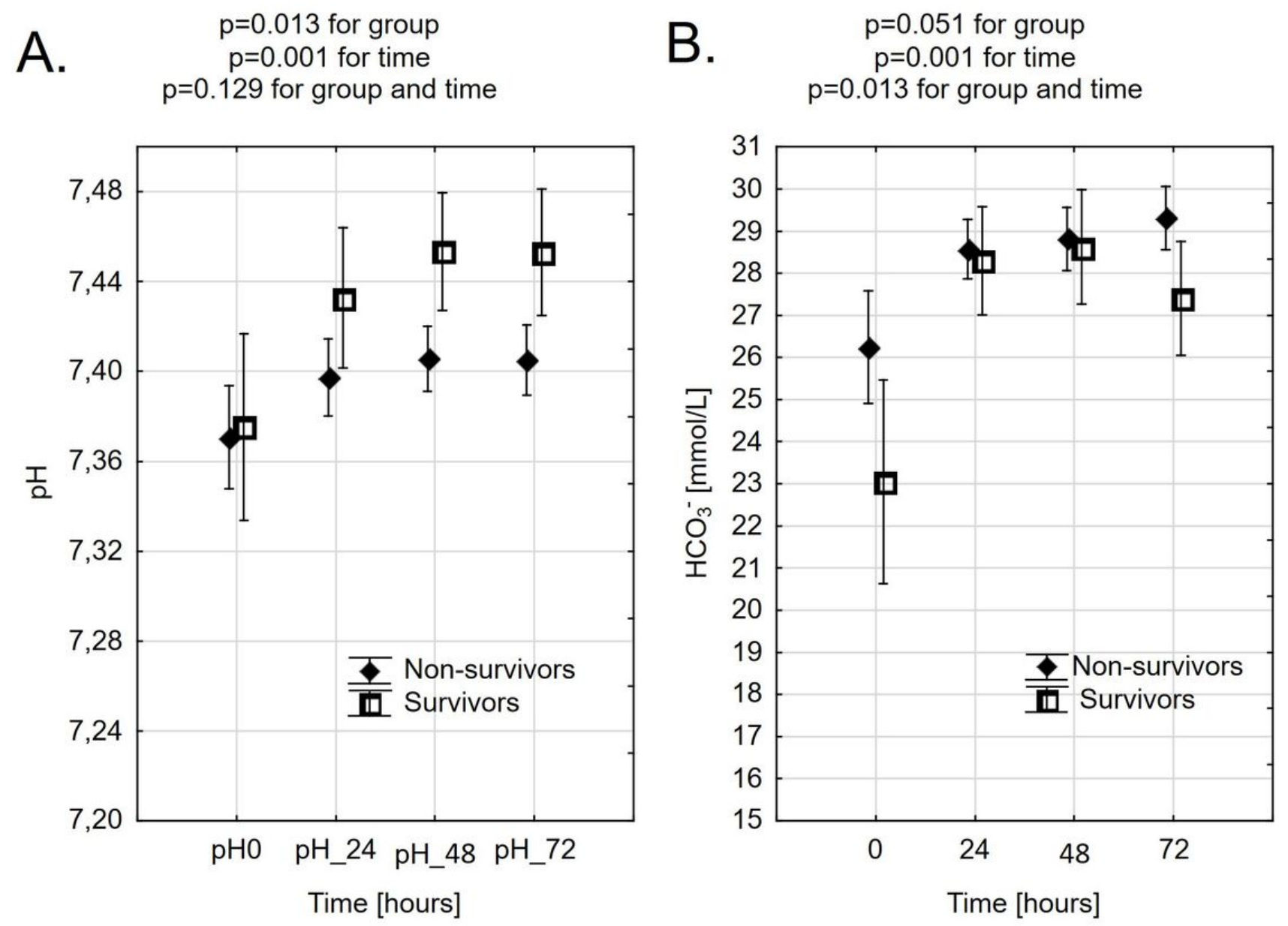

Figure 5

The $\mathrm{pH}$ values $(\mathrm{A})$ and $\mathrm{HCO}$ - concentrations $(\mathrm{B})$ during sessions longer than 72 hours in survivors and non-survivors. (means $\pm \mathrm{SD}$ ) 

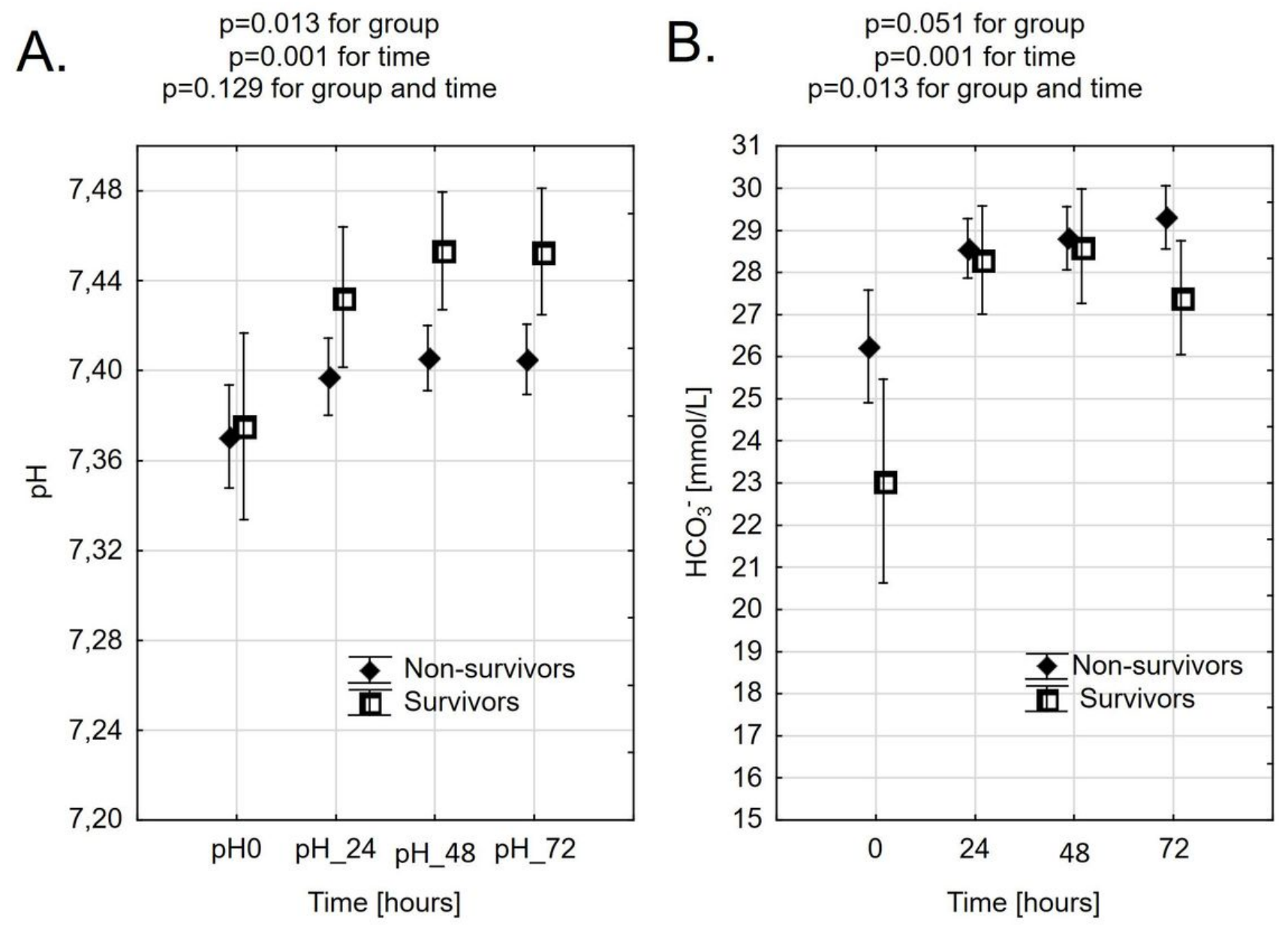

Figure 5

The $\mathrm{pH}$ values $(\mathrm{A})$ and $\mathrm{HCO}$ - concentrations $(\mathrm{B})$ during sessions longer than 72 hours in survivors and non-survivors. (means $\pm \mathrm{SD}$ ) 

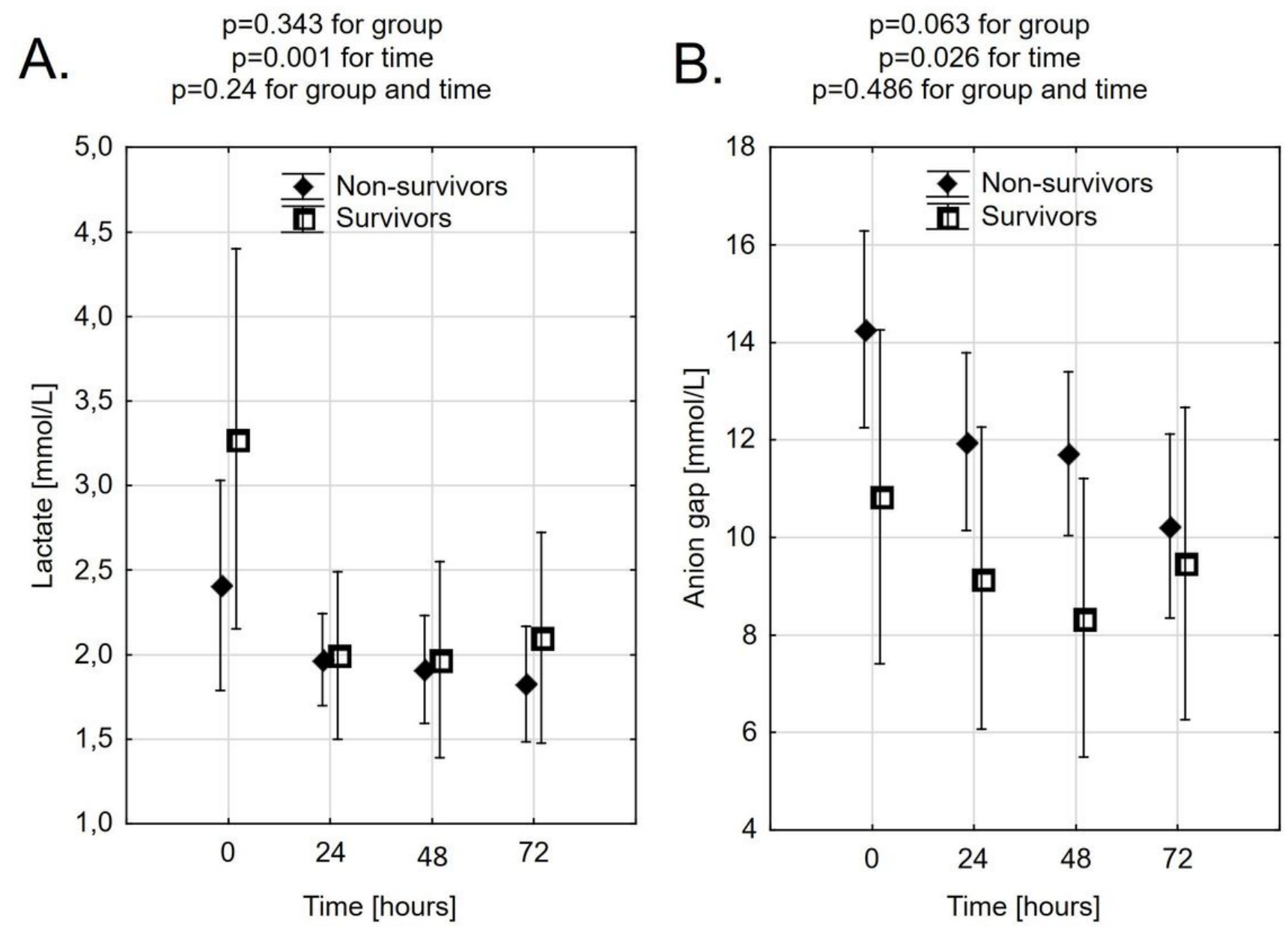

Figure 6

Lactate concentrations (A) and anion gaps (calculated as $\mathrm{Na}+-(\mathrm{Cl}-+\mathrm{HCO}-))$ (B) during sessions longer than 72 hours in survivors and non-survivors. (means $\pm S D)$ 

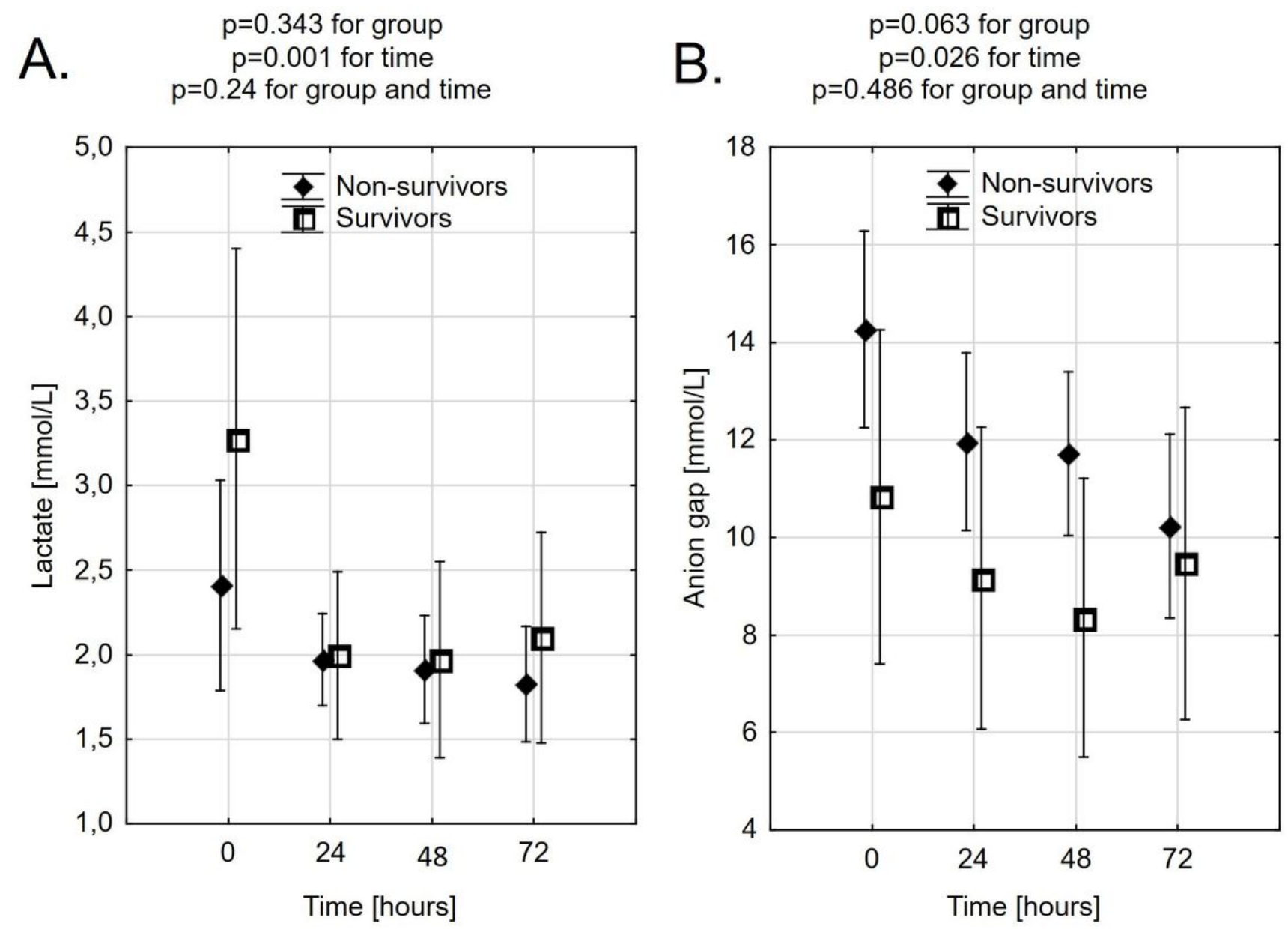

Figure 6

Lactate concentrations (A) and anion gaps (calculated as $\mathrm{Na}+-(\mathrm{Cl}-+\mathrm{HCO}-))$ (B) during sessions longer than 72 hours in survivors and non-survivors. (means $\pm S D)$ 

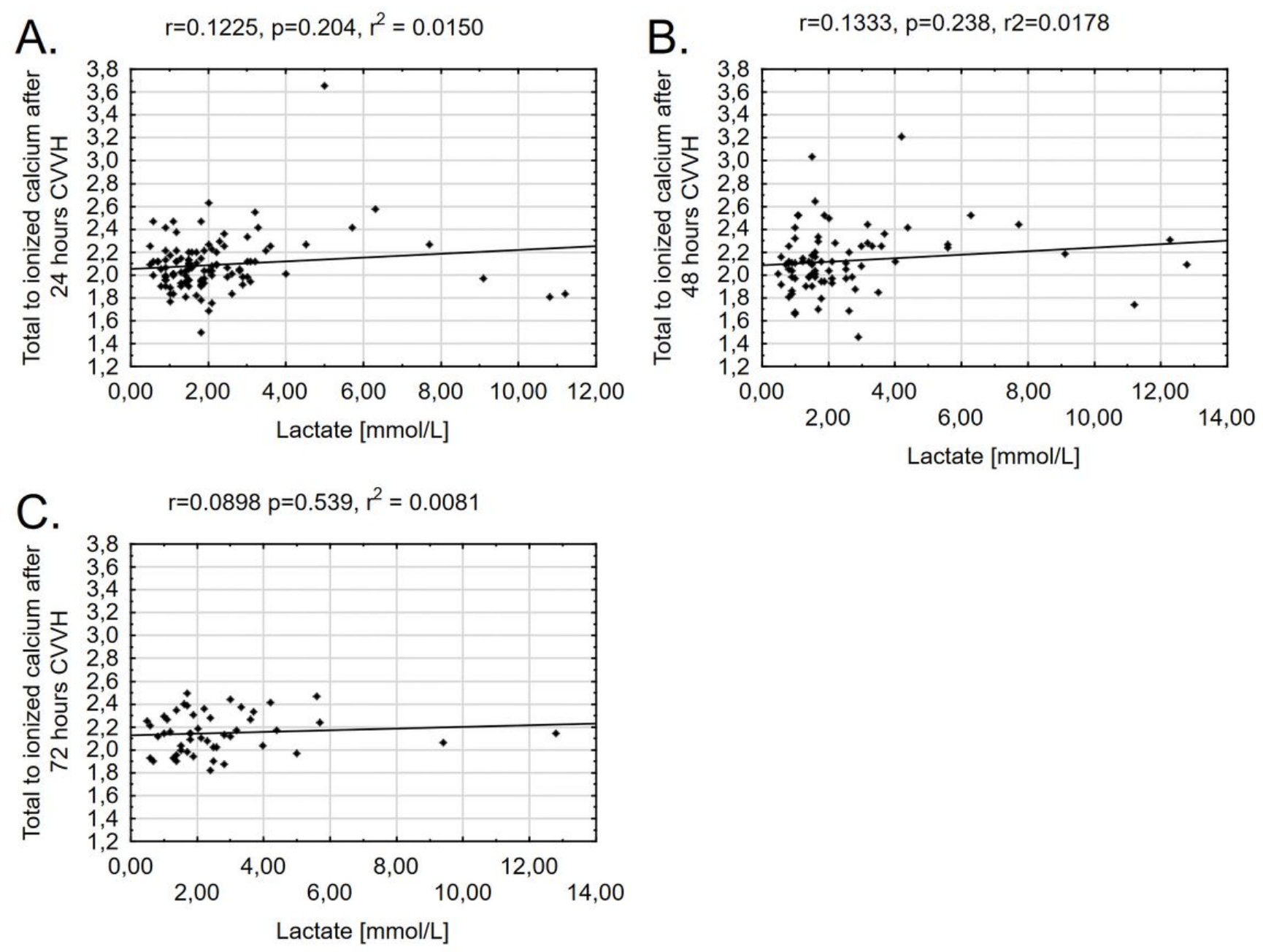

Figure 7

Correlation between the lactate concentration before the beginning of the haemofiltration session and the total to ionized calcium ratio after 24 ( $A$ ), 48 (B), and 72 (C) hours of haemofiltration. 

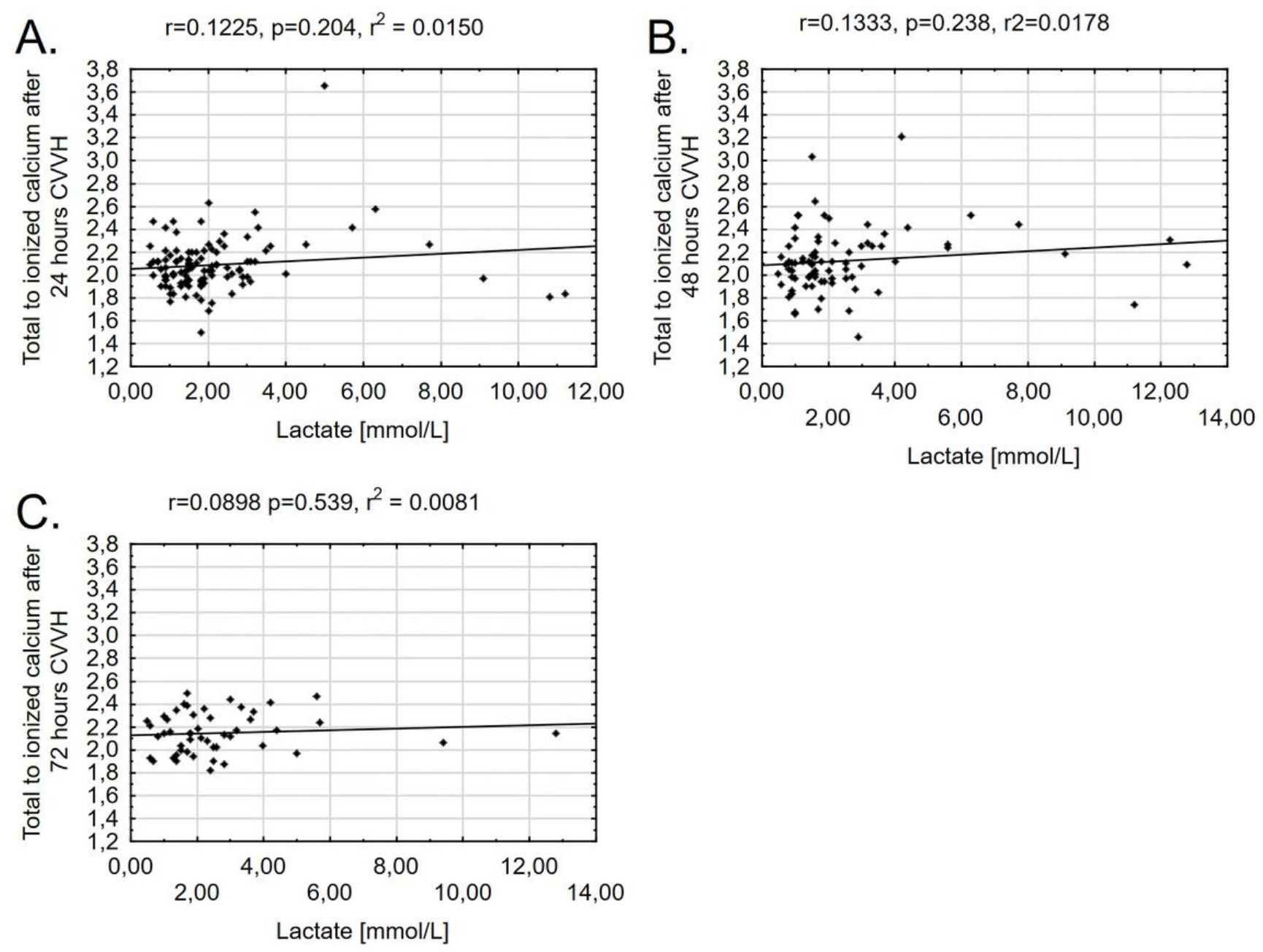

Figure 7

Correlation between the lactate concentration before the beginning of the haemofiltration session and the total to ionized calcium ratio after 24 ( $A$ ), 48 (B), and 72 (C) hours of haemofiltration.

\section{Supplementary Files}

This is a list of supplementary files associated with this preprint. Click to download.

- GB.JPG

- GB.JPG 